\title{
Long-term spectroscopic monitoring of the Luminous Blue Variable AG Carinae ${ }^{\star}$
}

\author{
O. Stahl ${ }^{1}$, I. Jankovics ${ }^{2}$, J. Kovács ${ }^{2}$, B. Wolf ${ }^{1}$, W. Schmutz ${ }^{3}$, A. Kaufer ${ }^{4}$, Th. Rivinius ${ }^{4}$, and Th. Szeifert ${ }^{4}$ \\ 1 Landessternwarte Königstuhl, 69117 Heidelberg, Germany \\ 2 Gothard Astrophysical Observatory, 9707 Szombathely, Hungary \\ 3 PMOD/WRC, 7260 Davos Dorf, Dorfstrasse 33, Switzerland \\ 4 European Southern Observatory, 85748 Garching, Karl-Schwarzschild-Str. 2, Germany
}

Received 10 April 2001 / Accepted 22 May 2001

\begin{abstract}
We have extensively monitored the Luminous Blue Variable AG Car (HD 94910) spectroscopically. Our data cover the years 1989 to 1999. In this period, the star underwent almost a full S Dor cycle from visual minimum to maximum and back. Over several seasons, up to four months of almost daily spectra are available. Our data cover most of the visual spectral range with a high spectral resolution $(\lambda / \Delta \lambda \approx 20000)$. This allows us to investigate the variability in many lines on time scales from days to years. The strongest variability occurs on a time scale of years. Qualitatively, the variations can be understood as changes of the effective temperature and radius, which are in phase with the optical light curve. Quantitatively, there are several interesting deviations from this behaviour, however. The Balmer lines show P Cygni profiles and have their maximum strength (both in equivalent width and line flux) after the peak of the optical light curve, at the descending branch of the light curve. The line-width during maximum phase is smaller than during minimum, but it has a local maximum close to the peak of the visual light curve. We derive mass-loss rates over the cycle from the $\mathrm{H} \alpha$ line and find the highest mass loss rates $\left(\log \dot{M} /\left(M_{\odot} \mathrm{yr}^{-1}\right) \approx-3.8\right.$, about a factor of five higher than in the minimum, where we find $\left.\log \dot{M} /\left(M_{\odot} \mathrm{yr}^{-1}\right) \approx-4.5\right)$ after the visual maximum. Line-splitting is very commonly observed, especially on the rise to maximum and on the descending branch from maximum. The components are very long-lived (years) and are probably unrelated to similar-looking line-splitting events in normal supergiants. Small apparent accelerations of the components are observed. The change in radial velocity could be due to successive narrowing of the components, with the absorption disappearing at small expansion velocities first. In general, the linesplitting is more likely the result of missing absorption at intermediate velocities than of excess absorption at the velocities of the components. The HeI lines and other lines which form deep in the atmosphere show the most peculiar variations. The HeI lines show a central absorption with variable blue- and red-shifted emission components. Due to the variations of the emission components, the HeI lines can change their line profile from a normal P Cyg profile to an inverse P Cyg-profile or double-peak emission. In addition, very broad $\left( \pm 1500 \mathrm{~km} \mathrm{~s}^{-1}\right)$ emission wings are seen at the strongest HeI lines of AG Car. At some phases, a blue-shifted absorption is also present. The central absorption of the HeI lines is blue-shifted before and red-shifted after maximum. Possibly, we directly see the expansion and contraction of the photosphere. If this explanation is correct, the velocity of the continuum-forming layer is not dominated by expansion but is only slightly oscillating around the systemic velocity.
\end{abstract}

Key words. stars: individual: AG Car - stars: early-type - stars: emission-line - stars: variable - stars: mass-loss

\section{Introduction}

The Luminous Blue Variables (LBVs) are a small group of hot and massive stars, characterized by pronounced variability on time-scales of years. Smaller variations at shorter timescales from weeks to months are also observed.

Send offprint requests to: O. Stahl,

e-mail: 0.Stahl@lsw.uni-heidelberg.de

* Based on observations collected at the European Southern Observatory at La Silla, Chile.
The LBVs are also known as S Dor variables after one of their prototypes objects. A few members of this group, such as $\eta$ Car and P Cyg, have shown so-called giant outbursts. More common are less extreme, but still very spectacular variations in spectral type (and $T_{\text {eff }}$ ) and brightness. The timescale of these variations is of the order of years and the typical amplitude in $V$ is $1-3$ magnitudes. The corresponding changes in spectral type are typically from late $\mathrm{O}$ - to intermediate A-supergiants. We will call 
these variations S Dor cycles in the following, in order to distinguish them from the rare giant outbursts.

During S Dor cycles, the color of the stars becomes redder with increasing visual brightness (van Genderen 1979), indicating lower temperature at maximum. The spectra behave in a similar way: the stars typically show A-supergiant spectra at visual maximum (Stahl \& Wolf 1982), while at minimum the spectra indicate B supergiants or late Of/WN stars. At all phases, the spectra show evidence for strong mass-loss. The more luminous objects seem to be hotter than the less luminous ones (Wolf 1989). This means that they populate at minimum an inclined instability strip in the Hertzsprung-Russell diagram, while at maximum they line up at the same temperature, but at different luminosities (Wolf 1989). The variations seem to occur at roughly constant bolometric magnitude (van Genderen 1979; Stahl et al. 1983; Viotti et al. 1984; Shore et al. 1996). Since the bolometric correction at maximum is very small, this means that the amplitude in visual light is a measure of the bolometric correction at minimum.

The constancy of bolometric luminosity implies strong changes in radius and temperature over the S Dor cycle. The reason for this radius increase is still unclear. While it has been suggested that the observed increase in the mass-loss rate during visual maximum (Wolf et al. 1981) changes the apparent stellar radius and effective temperature sufficiently to account for this (Wolf et al. 1981; Davidson 1987), it was found later that the observed mass-loss rates are far too low (Leitherer et al. 1989; de Koter et al. 1996; de Koter 1997) to explain the radius changes, at least for the "normal" $\mathrm{S}$ Dor maxima. It is even not clear that an increase in mass-loss rate is a general property of S Dor maxima (Stahl et al. 1990; Leitherer et al. 1994; Leitherer 1997). A sub-photospheric mechanism seems to be required to produce the radius increase. Candidates for this mechanism are e.g. cyclic outbursts during a phase of dynamical instability (Stothers \& Chin 1995; Stothers 1999) or "pulsation-triggered LBV eruptions" (Guzik et al. 1998). While the first mechanism is consistent with the observed time scales of years to decades (Stothers \& Chin 1995), the second possibility does not currently predict a time scale. We consider that the cause of LBV cycles is still unclear.

The main problem with observational studies of LBVs are the long time scales involved and the scarcity of bright objects suitable for detailed long-term studies. AG Car (HD 94910) - a galactic object with a visual magnitude between 6 and 8 -is relatively bright and possibly the LBV best suited for such a study. In contrast to P Cyg, which is the visually brightest LBV, it has been very active in recent decades. The well-known spectacular object $\eta$ Car is also very bright, but it is not representative of its group. Therefore, as the brightest active LBV, AG Car is the prototype object for LBVs.

The star AG Car has been the subject of several detailed studies. A century of photometric observations of AG Car was investigated by van Genderen et al. (1997).
One of the last maxima, which occurred in the early 1980's, has been studied by Wolf \& Stahl (1982) and Whitelock et al. (1983). Leitherer et al. (1994) studied in detail the early phase of the present maximum, which started in the late 1980's. Recent photometry was presented by Spoon et al. (1994). A spectroscopic study of the minimum phase around 1985 was published by Stahl (1986). The spectra of AG Car in 1985 resembles the Ofpe/WN9 stars. The reddening has been determined from IUE spectra to $E_{B-V}=0.65$ (Shore et al. 1996). This results in a luminosity of $L=10^{6 \pm 0.2} L_{\odot}$ (Shore et al. 1996; Leitherer et al. 1994) when a distance of $6 \pm 1 \mathrm{kpc}$ (Humphreys et al. 1989; Hoekzema et al. 1992) is used. Schulte-Ladbeck et al. (1994) presented spectropolarimetry of AG Car and found evidence for an axisymmetric stellar wind.

AG Car is surrounded by a well-known ring nebula (Thackeray 1950, 1977), which is probably the remnant of previous mass-loss events. Its morphology and kinematic properties have been studied in detail by Nota et al. (1995). A detailed abundance analysis of the nebula was published by Smith et al. (1997).

Despite these numerous studies of AG Car, no detailed spectroscopic study of the full activity cycle exists. The present paper intends to fill this gap.

\section{Observations}

AG Car was observed with several different echelle spectrographs and several telescopes at ESO, La Silla, from 1989, January to 1999, August. In the years 1989-1992 only snapshot observations were taken. The maximum phase between 1993 and 1999 is covered by yearly monitoring campaigns, each spanning several months. The epochs of observations and instruments used are summarized in Tables 1 and 2 .

Most of the observations were obtained with the fiberlinked echelle spectrographs FLASH (Mandel 1988) and its modified version Heros (Stahl et al. 1996), which were built at the Landessternwarte Heidelberg. These instruments were mainly used at the ESO $50 \mathrm{~cm}$ telescope, but for a few shorter runs also at the ESO $1.52 \mathrm{~m}$ and $2.2 \mathrm{~m}$ telescopes. Further observations with significantly better $S / N$ and resolution were carried out with Feros (Kaufer et al. 2000), also built at the Landessternwarte. This instrument was used at the ESO $1.52 \mathrm{~m}$ telescope during the commissioning phase and in the guaranteed observing time. In addition, a few spectra have been obtained with the CASPEC echelle spectrograph at the $3.6 \mathrm{~m} \mathrm{ESO}$ telescope.

In addition to these spectra, a few spectra obtained with the CEs-Instrument at the CAT-telescope at ESO, La Silla and at the Brazilian National Observatory (LNA) have been used. The spectra used are listed in Table 3 . For more details about these spectra see Leitherer et al. (1994).

In the FLASH configuration, a spectral range of about $2700 \AA$ was observed in one exposure. With the setting of 
Table 1. Summary of Flash, Heros and Feros observations.

\begin{tabular}{llrl}
\hline Instr. & Telescope & Sp. & MJD dates \\
\hline FLASH & ESO $50 \mathrm{~cm}$ & 1 & 48822 \\
FLASH & ESO $50 \mathrm{~cm}, 2.2 \mathrm{~m}$ & 93 & $49016-49139$ \\
FLASH & ESO $50 \mathrm{~cm}$ & 50 & $49386-49534$ \\
HEROS & ESO $50 \mathrm{~cm}$ & 43 & $49749-49871$ \\
HEROS & ESO $50 \mathrm{~cm}$ & 20 & $50105-50245$ \\
HEROS & ESO $50 \mathrm{~cm}, 1.52 \mathrm{~m}$ & 37 & $50449-50565$ \\
FEROS & ESO $1.52 \mathrm{~m}$ & 18 & $51135-51198$ \\
HEROS & ESO $50 \mathrm{~cm}$ & 13 & $51301-51365$ \\
FEROS & ESO $1.52 \mathrm{~m}$ & 4 & $51372-51392$ \\
\hline
\end{tabular}

the spectrograph used, the $\mathrm{H} \delta$ and $\mathrm{HeI} \lambda 6678$ lines could be observed with one exposure, i.e. the spectral range covered the wavelengths from $4050 \AA$ to $6780 \AA$. Heros is a modified version of FLASH, where a beam splitter is used to divide the beam after the echelle grating into two channels, each with its own cross-disperser, camera and CCD detector. The red channel is identical to the FLASH instrument. The two spectral ranges of the HERos configuration cover the range from $3450 \AA$ to $5560 \AA$ in the blue channel and from $5820 \AA$ to $8620 \AA$ in the red channel. The spectral resolution is about 20000 for both FLASH and Heros. The $S / N$-ratio of our spectra strongly depends on the wavelength and is lowest in the blue spectral range. For AG Car we typically used an exposure time of one to two hours for FLASH and HEROs with the ESO $50 \mathrm{~cm}$ telescope. In good conditions, a $S / N$-ratio of at about 100 is reached in the red spectral range.

The FERos spectra cover the spectral range from about $3600 \AA$ to $9200 \AA$ in 39 orders. The spectral resolution of Feros is about 48000 . The $S / N$ is much higher than for the FLASH and HEROs spectra. It depends strongly on wavelength and is highest in the red spectral region. The typical exposure times were about $10 \mathrm{~min}$.

The CAspec observations are summarized in Table 2. The data have been obtained in different settings and with different detectors available at the time of observation.

For all instruments, a built-in high-temperature incandescent lamp and a ThAr lamp were used to obtain flatfield and wavelength-calibration exposures, respectively.

All spectra were reduced with ESO-Midas. The CASPEC spectra were reduced with the standard echelle package of ESO-Midas (Ponz \& Brinks 1986). For the FLASH and HEROs spectra a modified version of this package (Stahl et al. 1993) was used. The FERos package (Stahl et al. 1999), also running within ESO-Midas, was used for the reduction of the FEROs spectra.

In the monitoring programs, the star was observed in intervals of at least once per week and up to once per night in some seasons. In these runs, the sampling is sufficiently dense to cover variations with time scales of a few days.

Our data cover most of the last maximum phase of AG Car. The lightcurve of AG Car is shown in Fig. 1. It has been constructed from visual observations of amateur observers that have been published by the RASNZ (Bateson 1988-2000). Although these observations are not
Table 2. Summary of CASPEC observations. The spectral resolution is about 20000 .

\begin{tabular}{cc}
\hline Spectral range & MJD date \\
\hline $3900-4900 \AA$ & 47549 \\
$3800-5300 \AA$ & 48285 \\
$3900-5300 \AA$ & 48609 \\
$3800-5300 \AA$ & 49342 \\
$3600-5000 \AA$ & 50771 \\
$5400-6700 \AA$ & 50771 \\
\hline
\end{tabular}

Table 3. Summary of CES and LnA observations. The spectra cover the region around $\mathrm{H} \alpha$ with a spectral resolution of 60000 and 8000 , respectively.

\begin{tabular}{ccc}
\hline MJD date & instrument & Number of spectra \\
\hline 48469 & CES & 1 \\
48624 & CES & 2 \\
48698 & CES & 2 \\
48757 & CES & 3 \\
49132 & CES & 2 \\
\hline 48247 & LNA & 1 \\
48483 & LNA & 1 \\
48557 & LNA & 1 \\
\hline
\end{tabular}

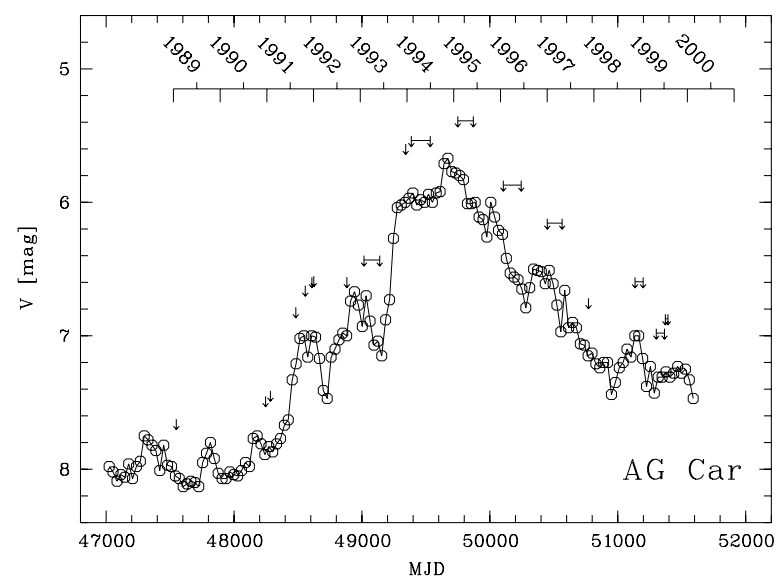

Fig. 1. Lightcurve of AG Car as derived from the observations published in the circular of RASNZ (Bateson 1988-2000). The dates and ranges, where spectroscopic observations have been obtained, are marked.

of photoelectric quality, we used them since this is the most complete photometric dataset available. The dates of our spectroscopic observations are marked in the figure. It can be seen that the spectra cover most of the maximum phase of the recent $\mathrm{S}$ Dor cycle of AG Car.

Part of the data discussed in this paper have already been presented at several meetings (Stahl 1997, 1998, 2001).

\section{Long-term variability}

\subsection{Variability of effective temperature and radius}

For the following discussion of the spectral variability, it is useful to have an estimate of effective temperature and 
radius over the S Dor cycle for all dates. This information can be extracted from the lightcurve, if we assume a constant bolometric luminosity (Viotti et al. 1984; Shore et al. 1996).

We follow the procedure of Schmutz (1997), who used two theoretical models to calibrate empirically the relation of the astrophysical flux in the $V$-band to the effective temperature. The relation implicitly assumes that the bolometric luminosity is constant for all models. With this relation, the temperature and radius can be computed for any given visual magnitude. Schmutz (1997) used only two models to calibrate the relation and therefore could only derive a linear relation. Since both models were relatively hot, the relation cannot be expected to work very well for cooler models. Using additional models (see below), we refined the relation by including a non-linear term.

$\log F_{5555}=-53.10+21.83 \log T-2.37(\log T)^{2}$.

Here $F_{5555}$ is the astrophysical flux emitted at $5555 \AA$ expressed in erg cm $\mathrm{cm}^{-2} \mathrm{~s}^{-1} \mathrm{~Hz}^{-1}$ and $T$ is in $\mathrm{K}$. The relation is valid approximately for $3.9<\log T<4.4$ and only for AG Car. In particular, we note that the derived fluxes are a function of the mass-loss rate and the adopted velocity law. Therefore, we get different $F_{5555}$ values for the same temperature but different mass-loss rates (see Sect. 4). The given relation results from a least squares fit to the model results.

Figure 2 illustrates the dramatic changes of $T_{\text {eff }}$ and $R_{*}$ over the $\mathrm{S}$ Dor cycle. The radius varies from about 50 to $500 R_{\odot}$ and the effective temperature from about 26000 to $8000 \mathrm{~K}$.

The result is not strongly dependent on this calibration of Eq. (1), however. We calculated the effective temperature and radius also by using the black-body approximation to compute the astrophysical flux. We then obtain quite similar results. It should be noted that the bolometric luminosity of LBVs is possibly not completely constant during maximum. Lamers (1995) found that the bolometric luminosity decreases slightly during maximum. A possible reason for this drop in the star's luminosity is the work needed to lift the outer layers. This change in $M_{\text {bol }}$ is neglected here.

\subsection{Change of spectral appearance}

As expected from the very remarkable change in $T_{\text {eff }}$, the spectroscopic changes are very strong. This is illustrated for selected parts of the spectrum in Fig. 3. In general, the spectral evolution of the star follows the lightcurve very closely. At minimum, the spectrum resembles Ofpe/WN9 stars (Stahl 1986), with HeII $\lambda 4686$ in emission and many HeI lines showing $\mathrm{P}$ Cygni profiles (Figs. 3b and c). Forbidden emission lines of [FeIII] are also present at minimum brightness (Fig. 3a).

HeII $\lambda 4686$ and the forbidden [FeIII] lines disappear very rapidly after the onset of the rise to maximum. In this phase, the spectral appearance is very similar to the spectrum of P Cyg (Stahl et al. 1993), i.e. the spectral

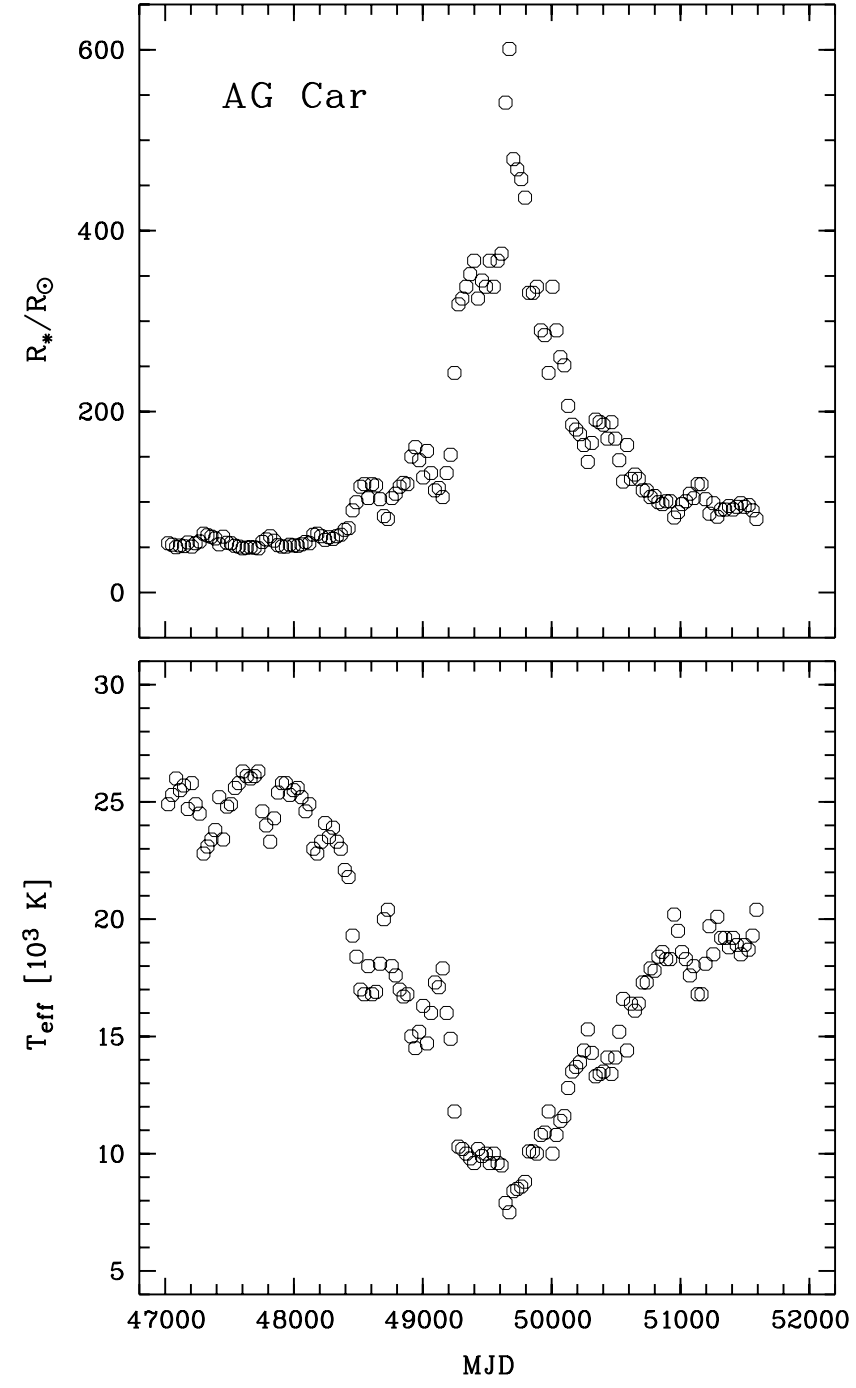

Fig. 2. Radius and effective temperature versus date for AG Car computed from the photometric data (Bateson 1988-2000) given in Fig. 1. For details see text.

type is early B. About one magnitude above minimum brightness, [FeII] lines become visible (Fig. 3a). HeI lines are already quite weak at that brightness. $[\mathrm{NII}] \lambda 5755$ is also seen at intermediate brightness levels.

Close to maximum, the spectrum is dominated by lines of singly ionized metals, especially by FeII and Tiri lines. The spectrum resembles an early-A hypergiant, but with much stronger emission. It is very similar to the maximum spectrum of other LBVs, e.g. S Dor (Leitherer et al. 1985). Most of the metal lines also show pronounced P Cygni profiles. The only exception is MgII $\lambda 4481$, which shows a weak P Cygni profile near minimum light, but only a very strong absorption feature appears around maximum. Close to the visual maximum, the NaI $\lambda \lambda 5890,5895$ resonance doublet also shows a clear P Cygni profile. At the very peak of the visual light curve, the lines from other singly ionized metals - more temperature sensitive than the FeII lines, e.g. CrII and TiII - also become strong. After the maximum the evolution is reversed. CrII and TiII lines disappear first, later on also the FeII lines. While the 

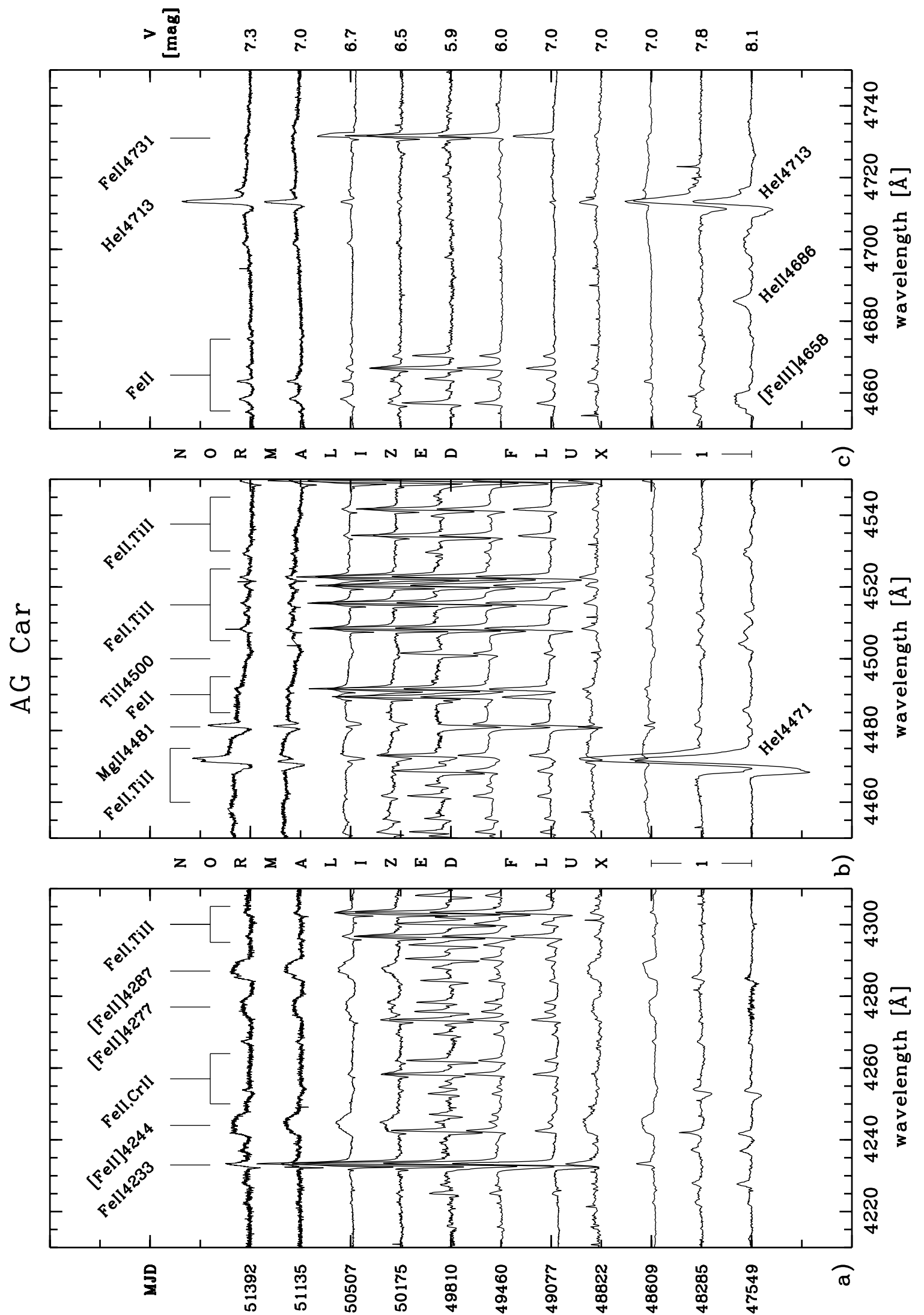

Fig. 3. Long-term spectral changes in the region around the [FeII] $\lambda \lambda 4244,4277,4287$ lines [panel a)], HeI $\lambda 4471$ line [panel b)], and HeII $\lambda 4686$ line [panel c)]. MJD of the observations, the scale of the normalized flux and the estimated visual brightness of the star at the given MJD are also indicated. 


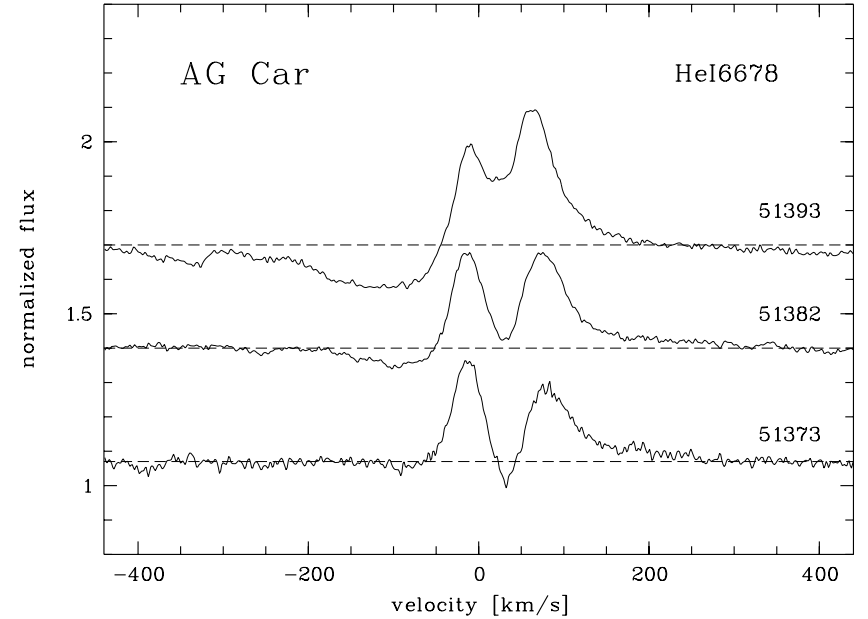

Fig. 4. Re-appearing of the high-velocity wind in the HeI $\lambda 6678$ line and simultaneous weakening of the low-velocity absorption in 1999. Numbers indicate the MJD of the exposure. Dashed lines represent the continuum level.

visual brightness declines, the [FeII] lines become visible and later, about one magnitude below maximum, the HeI lines with strong P Cyg profiles appear again. The spectral appearance of the star becomes similar to the spectra observed about one magnitude below maximum on the rising branch.

The re-appearance of the HeI $\lambda 6678$ feature is very spectacular. The "un-shifted" absorption component of the double peaked profile disappears rapidly (between MJD $=51373$ and 51393, Fig. 4). The re-appearance of HeII $\lambda 4686$ after maximum has not been observed in our spectra. This is not surprising, since the star was still far from minimum.

Overall, the observations indicate that the visual brightness is a good indicator of the effective temperature of the expanded photosphere during maximum. The excitation and ionization of the stellar-wind lines follow the visual brightness very closely.

One of the peculiarities of the maximum spectra is that the HeI lines never disappear completely. While they show typical P Cyg profiles around minimum, they have very peculiar profiles at the bright phases of the star. They appear to show two absorption components - one of them sometimes red-shifted - and a central emission component or two emission components. Examples are shown in Fig. 20. MgII $\lambda 4481$ shows similar profiles during phases of intermediate brightness.

\subsection{Stellar-wind velocity}

The systemic velocity $v_{\text {sys }}$ is needed to convert the measured velocities to the rest frame of the star. For AG Car, this quantity is also of special interest, since it has been used for an estimate of its kinematic distance (Humphreys et al. 1989). Since the distance of AG Car is very important for the interpretation of all observations, we rederived the systemic velocity from our spectra.

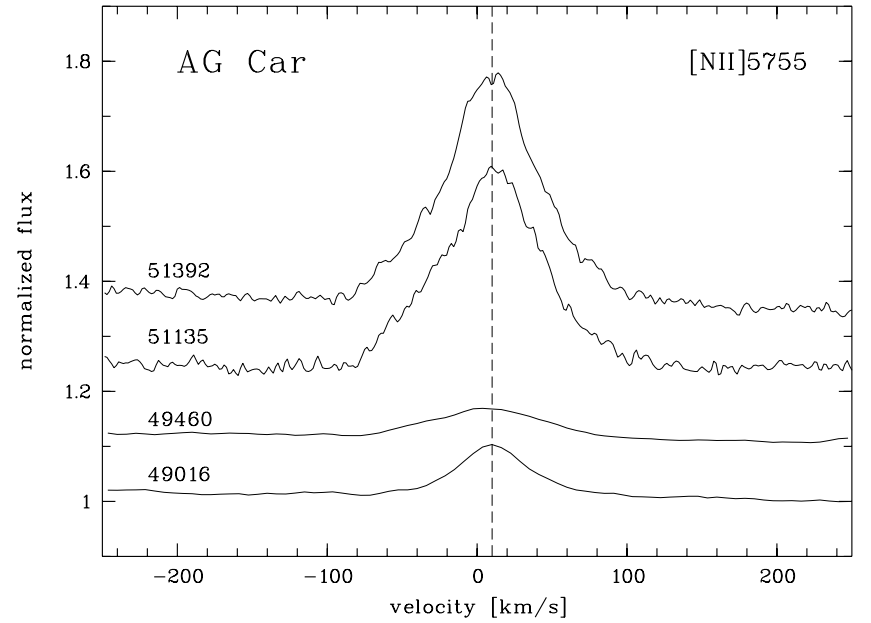

Fig. 5. The line profile of $[\mathrm{NII}] \lambda 5755$ for various phases.

Unfortunately, the systemic velocity is difficult to measure accurately from a complicated emission spectrum such as the spectrum of AG Car. As a forbidden line, [NII] $\lambda 5755$ is not affected by absorption and it is very symmetric. Therefore it may be the line best suited for the determination of the systemic velocity. It forms at relatively high densities and therefore does not originate in the nebula but probably in the outer stellar wind. Using a laboratory wavelength of $5754.57 \AA$ (Bowen 1960) for this line, we determined a heliocentric radial velocity from our FEROs spectra (taken after maximum) of about $9 \pm 3 \mathrm{~km} \mathrm{~s}^{-1}$. This line is also weakly present on our FLASH spectra taken before maximum, where it gives the same value for $v_{\text {sys }}$, but it falls in the gap between both channels in the Heros spectra. The profile of this line is shown in Fig. 5.

The forbidden [FeII] lines can also be used to derive the systemic velocity. These lines are also not affected by $\mathrm{P}$ Cygni absorption and are relatively symmetric (see Fig. 6). They are broader and weaker than [NII] $] 55755$, however, and partly affected by blends. We find a heliocentric radial velocity of about $10 \mathrm{~km} \mathrm{~s}^{-1}$ from the bisector of these lines.

In addition, we used the HeII $\lambda 4686$ line, and a number of FeII, FeIII, AlıI and MgII lines which have weak absorption components, to check this value. All these lines are consistent with a heliocentric systemic velocity of $10 \pm 5 \mathrm{~km} \mathrm{~s}^{-1}$. The fitting of the $\mathrm{H} \alpha$ line, however, favors a higher systemic velocity of about $20 \mathrm{~km} \mathrm{~s}^{-1}$ (see Sect. 4). In the following we will assume a systemic velocity of $10 \mathrm{~km} \mathrm{~s}^{-1}$ for AG Car.

A heliocentric systemic velocity of $20 \pm 2 \mathrm{~km} \mathrm{~s}^{-1}$, based on measurement of the nebula and on the emission components of P Cygni profiles was used by Humphreys et al. (1989) to derive a kinematic distance of 6.4-6.9 kpc. Our value for the systemic velocity of $10 \mathrm{kms}^{-1}$ favors a smaller distance of about $5-6 \mathrm{kpc}$, but it is still compatible with the distance estimate of $6 \mathrm{kpc}$ derived by Humphreys et al. (1989) and Hoekzema et al. (1992). 


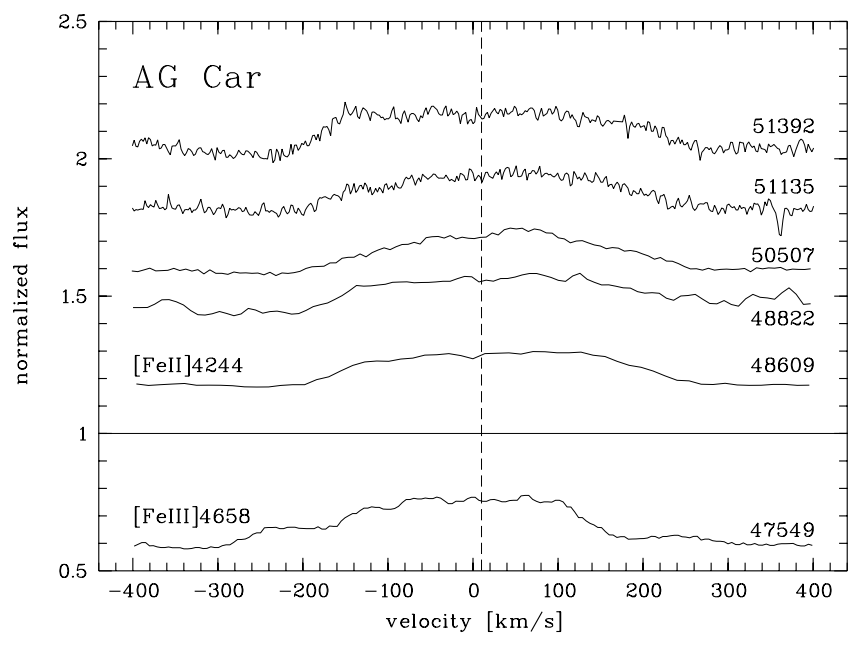

Fig. 6. Forbidden emission lines of the single ([FeII $] \lambda 4244$, upper part) and double ionized ([FeIII] $\lambda 4658$, lower part) iron. The systemic velocity of $10 \mathrm{~km} \mathrm{~s}^{-1}$ and MJDs of spectra are indicated.

Since the forbidden lines form at large distances from the star, the terminal stellar wind velocity $v_{\infty}$ can be derived from the width of these lines, assuming that the lines form in the stellar wind. Since the [FeII] lines are flattopped (Fig. 6), they are likely to be formed in a region of constant outflow velocity, i.e. their width directly measures the terminal velocity of the wind. Unfortunately, the [FeII] lines appear only at intermediate brightness levels. We find an expansion velocity of about $200 \mathrm{~km} \mathrm{~s}^{-1}$ both before and after maximum from these lines. Since the [FeII] lines are seen only for a limited time, they cannot be used to measure the terminal velocity at minimum and maximum. The [FeIII] lines, which appear at minimum, seem to have a complex profile (Fig. 6) and are therefore unsuitable for the determination of the stellar wind velocity.

The standard way to determine the stellar wind velocity is by measuring the blue edge of strong resonance lines with $\mathrm{P}$ Cyg profiles. Unfortunately, there are no resonance lines in the optical range which we could use for this purpose. Therefore we used the Balmer lines, which have strong absorption components. Since these lines often have no well defined blue edge, this measurement gives a lower limit to $v_{\infty}$. The absorption is not strong enough at large expansion velocities. The absorption is not black, so that this measurement can be affected by turbulence in the wind (Prinja et al. 1990). In this case, the edge velocity can be lower than $v_{\infty}$. Also, since the Balmer lines frequently show split absorption components, measurements of the edge velocity are difficult (Figs. 7 and 11).

Using the $\mathrm{H} \beta$ profiles we find that the approximate edge velocity is $-290 \mathrm{~km} \mathrm{~s}^{-1}$ during minimum and about $-140 \mathrm{~km} \mathrm{~s}^{-1}$ during maximum (Fig. 7). With $v_{\text {sys }}=$ $10 \mathrm{~km} \mathrm{~s}^{-1}$, the corresponding expansion velocities are 300 and $150 \mathrm{~km} \mathrm{~s}^{-1}$, respectively. During maximum, the edge velocity does not show pronounced variations. After the initial decrease from its higher value in minimum, the edge velocity of the Balmer lines is almost constant and basi-

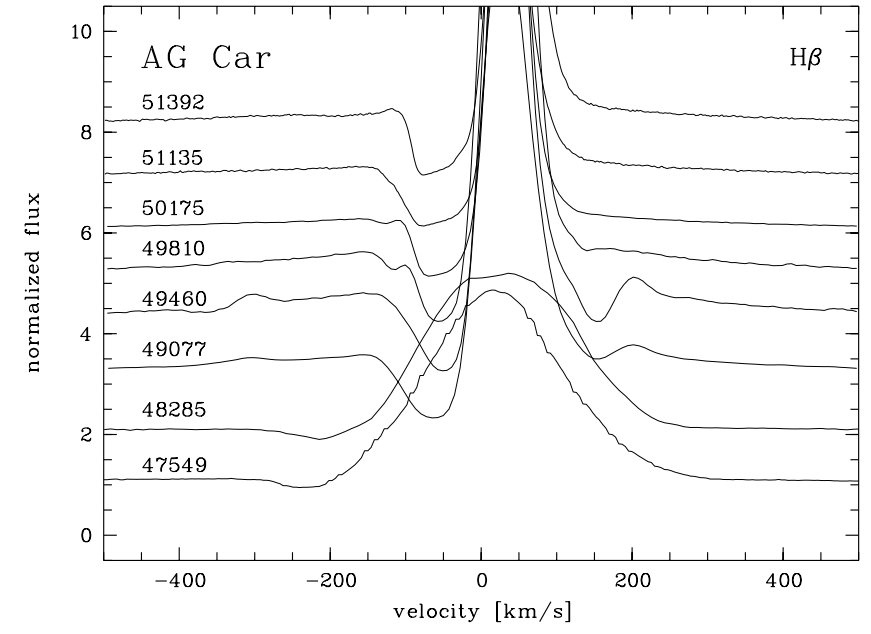

Fig. 7. Variability of the $\mathrm{H} \beta$ line over the full $\mathrm{S}$ Dor cycle. While the edge velocity is clearly variable, the edge is not well defined and difficult to measure exactly.

cally independent of visual brightness. This is true for a range of at least one magnitude in visual brightness. After the maximum, the edge velocity even appears to decrease significantly. Only a high velocity component extends to the velocity observed at maximum (Fig. 11, spectrum labeled 51393).

Another possible way to estimate $v_{\infty}$ is to measure the $F W H M$ of strong emission lines and to assume that the ratio of $F W H M$ to $v_{\infty}$ is constant with time. If this ratio can be calibrated, the measurement of the FWHM can be used to measure the variations of $v_{\infty}$. This approach has been used by Leitherer et al. (1994). In Fig. 16a the $F W H M$ of $\mathrm{H} \alpha$ is plotted versus time. It can be seen that the $F W H M$ of the line peaks during the rise to maximum and then starts to decline. The line width does not show a clear correlation with the edge velocity.

The Balmer lines have strong and very broad emission wings, which extend to more than $\pm 1500 \mathrm{~km} \mathrm{~s}^{-1}$ from line center (Fig. 11). Such wings were first found for LBVs in P Cygni by Bernat \& Lambert (1978). These wings are explained by scattering of line photon by free electrons in the stellar wind. In this model, the large line width reflects the high thermal velocities of the electrons (due to the small electron mass) and does not correspond to bulk motion of the gas. In order to produce strong emission wings, a strong emission-line core is needed. It is therefore surprising that at some phases, in particular during rise to maximum, also very weak HeI emission-lines have emission wings of considerable strength. A striking example is shown in Fig. 8. We note that similar wings to HeI lines were reported by Stahl et al. (1983) for the LBV R 127 of the LMC, also during the initial rise to maximum. Although the interpretation as electron scattering wings is not obvious for these HeI lines, we still believe that this is the most likely explanation. It requires, however, that the line photons producing these wings are almost completely scattered in the wings, i.e. the electron scattering optical depth must be very high. 


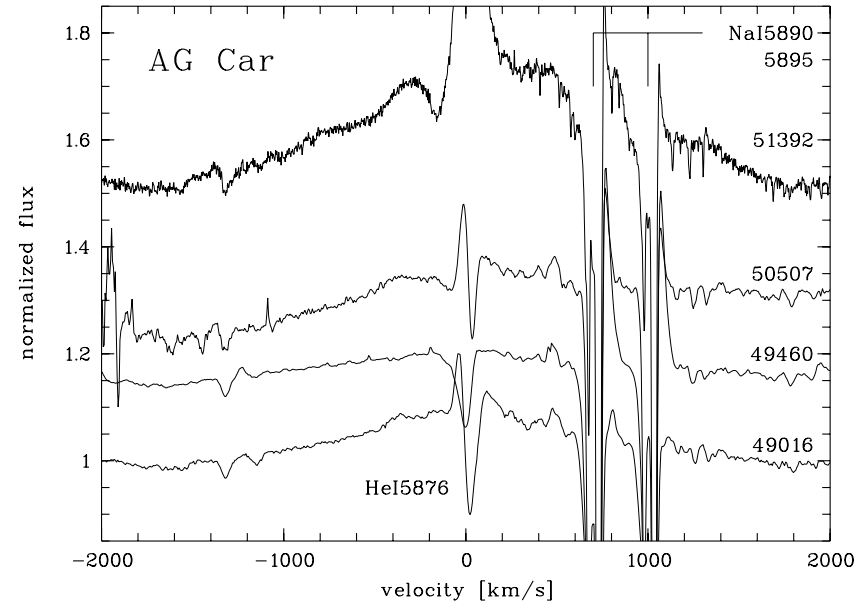

Fig. 8. Strong emission line wings around the HeI $\lambda 5876$ line in the spectrum of AG Car taken during the rise to maximum. The wings extend to about $\pm 1500 \mathrm{~km} \mathrm{~s}^{-1}$ from line center. The numbers denote the date of exposure in MJD.

\subsection{Absorption-line splitting}

Absorption-line splitting is observed in many early-type stars, but it is particularly pronounced in LBVs. E.g. in $\mathrm{R}$ 127, three components have been observed during the rise to maximum (Stahl et al. 1983).

In AG Car, the line splitting seems to be most pronounced in the rise to maximum and later again in the decline phase. Four absorption components have been observed in the $\mathrm{H} \alpha$ line of AG Car by Leitherer et al. (1994) in the early phase of the present S Dor cycle. Our observations extend over a longer time span than the observations by Leitherer et al. (1994) and we therefore can follow the evolution of these features in more detail. In the $\mathrm{H} \alpha$ line, the features are seen for a period of several years. The line splitting is not only visible in the Balmer lines but also in other wind lines, e.g. the stronger FeII lines.

The results are summarized in Table 4 . The features have a minimum lifetime of $(49139-48470)=669$ days. They are not yet seen in MJD $=48285$ (in $\mathrm{H} \beta$ ) and no longer at $\mathrm{MJD}=49386$, which means that their lifetime is less than 1100 days. One feature in HeI $\lambda 6678$ is even seen much longer (see below). The velocity of the fastest component corresponds well with the stellar wind velocity in minimum. This is demonstrated in Fig. 9, where the appearance of the components at the beginning of the rise to maximum is shown. The appearance of the components coincides with the rise from minimum (see Fig. 1).

From Table 4 and Fig. 9 it can be seen that the velocities of discrete components observed in $\mathrm{H} \alpha$ and $\mathrm{H} \beta$, except the main, slow component $\left(v_{1}\right)$, appear to increase slightly with time. The effect is very small, however, but the increase in $v_{2}$ seems to be significant.

Two components are also seen in CaII $\lambda \lambda 3933,3968$ around maximum. In Dec. 1993 they were first seen and reached maximum strength in 1995, close to the visual maximum light, and weakened later. The components were not visible in Dec. 1991 and in 1999. The features

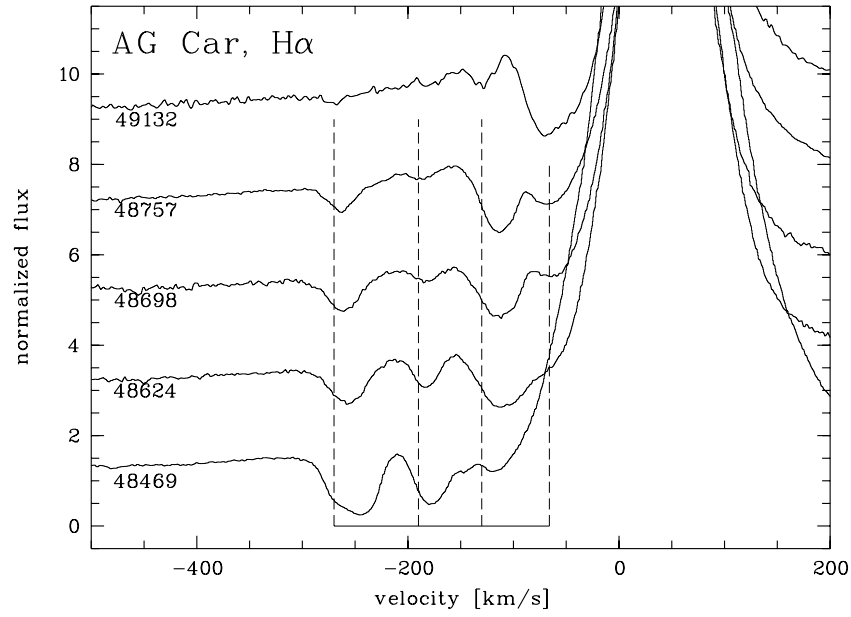

Fig. 9. Evolution of the absorption components in the $\mathrm{H} \alpha$ line from 1991 to 1993. Up to four components are visible. No splitting was seen in $\mathrm{H} \beta$ half a year before the first spectrum in this series. The spectra are marked with MJD. The vertical lines mark velocities of $-66,-130,-190$ and $-270 \mathrm{~km} \mathrm{~s}^{-1}$.

Table 4. Measured velocities of the discrete absorption components in $\mathrm{H} \alpha$ and $\mathrm{H} \beta$. Heliocentric velocities are given. A slight increase of the expansion velocity is indicated.

\begin{tabular}{lccccl}
\hline MJD & $v_{1}$ & $v_{2}$ & $v_{3}$ & $v_{4}$ & Ref. \\
\hline 48469 & & -119 & -176 & -252 & $\mathrm{H} \alpha$, CES \\
48609 & -66 & -110 & -190 & -266 & $\mathrm{H} \beta$, CASPEC \\
48624 & & -108 & -184 & -258 & $\mathrm{H} \alpha$, CES \\
48698 & & -114 & -187 & -263 & $\mathrm{H} \alpha$, CES \\
48757 & -68 & -113 & -189 & -264 & $\mathrm{H} \alpha$, CES \\
48822 & -71 & -119 & -192 & -268 & $\mathrm{H} \alpha$, FLASH \\
49016 & -67 & -125 & -188 & -269 & $\mathrm{H} \alpha$, FLASH \\
49132 & -63 & -132 & -200 & -271 & $\mathrm{H} \alpha$, CES \\
49139 & -65 & -132 & -188 & -270 & $\mathrm{H} \alpha$, FLASH \\
\hline
\end{tabular}

Table 5. Measured velocities of the discrete absorption components in CaII $\lambda 3933$. Heliocentric velocities are given. The velocities are, with the exception of the measurement $v_{1, \text { CaII }}$, at MJD 49342 almost constant.

\begin{tabular}{lcc}
\hline MJD & $v_{1, \text { CaII }}$ & $v_{2, \text { CaII }}$ \\
\hline 49342 & -141 & -201 \\
$49749-49871$ & -150 & -208 \\
$50105-50245$ & -152 & -209 \\
$50449-50565$ & -149 & -207 \\
\hline
\end{tabular}

here have no obvious counterpart in any other line. The evolution of these components $\left(v_{1, \text { CaII3933 }}, v_{2, \text { CaII3933 }}\right)$ is shown in Fig. 10. The velocities are summarized in Table 5. Similar to the components in the Balmer lines, the radial velocities are almost constant.

Line-splitting is also observed after the maximum. Here, for most of the time, only two components are seen. We could follow the faster component from 1996 to 1999 (at least 1287 days). The radial velocity of the fastest absorption component corresponds well with the stellar wind velocity in maximum. This is demonstrated in Fig. 11, where the appearance of the splitting after maximum is 


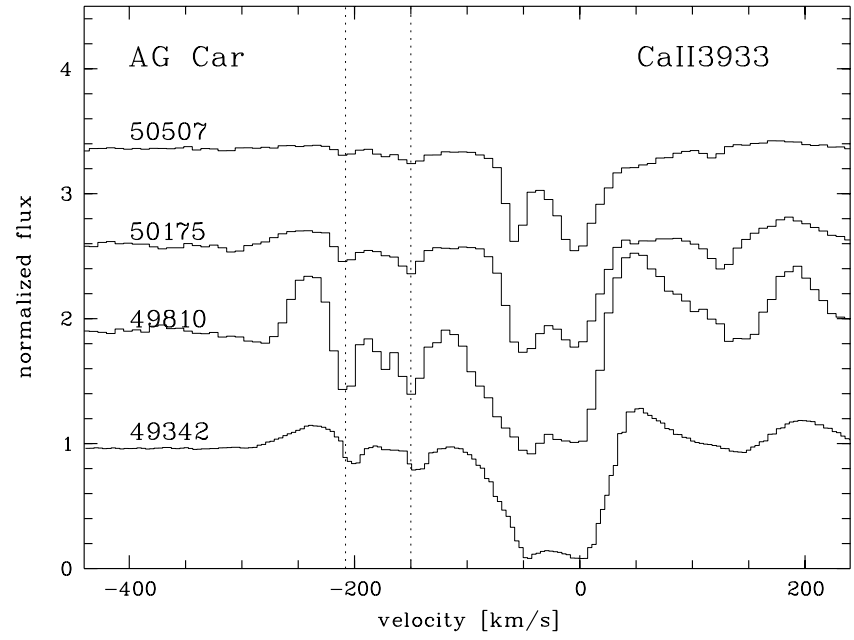

Fig. 10. Evolution of the absorption components in the line CaII $\lambda 3933$ from 1993 to 1997 . The lines mark velocities of -150 and $-208 \mathrm{~km} \mathrm{~s}^{-1}$, respectively.

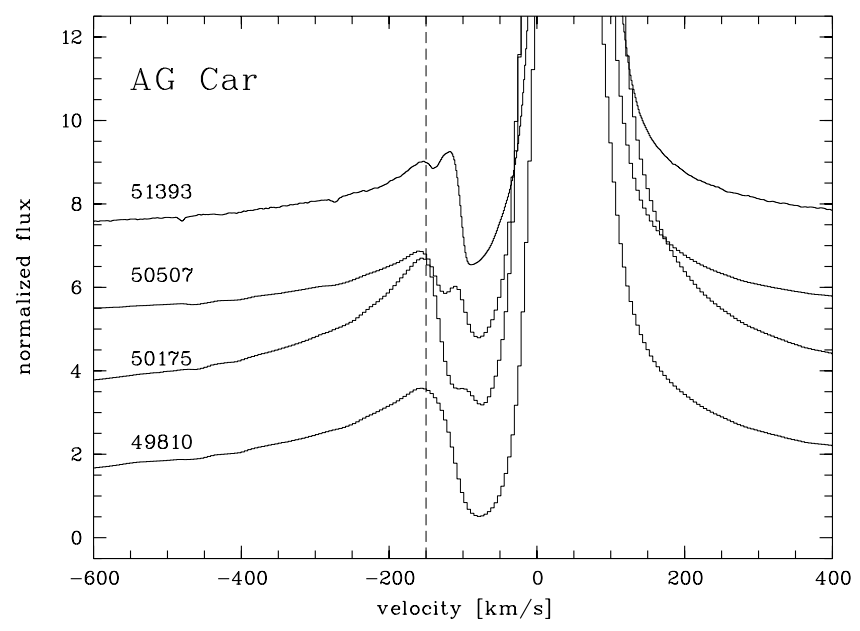

Fig. 11. Appearance of the absorption components in the $\mathrm{H} \alpha$ line from 1996 to 1999. In the first spectrum, taken in 1995, no components are seen. In 1996, a separated component appears which became fainter in the following years. The vertical line marks a heliocentric radial velocity of $-150 \mathrm{~km} \mathrm{~s}^{-1}$. The emission-line wings extend well beyond the spectral region shown here. The spectra are marked with MJD.

shown. This line-splitting event was covered better by our observations than the event at the beginning of the S Dor cycle.

We could follow the feature in several observing seasons from 1996 to 1999. Since the line components are better separated in the metal lines than in the Balmer lines, we show the evolution in velocity, line width, $F W H M$, and equivalent of the high-velocity component of FeII $\lambda 5169$ in Fig. 12. A small but significant acceleration is detected. This acceleration is accompanied by a decrease of line width and equivalent width. The mean profile of the line in the years 1995, 1996, 1997 and 1998/1999 is shown in Fig. 13. This figure illustrates that the edge velocity of the blue-shifted component $\left(-140 \mathrm{~km} \mathrm{~s}^{-1}\right)$ does not change. The apparent acceleration is due to the narrowing of the
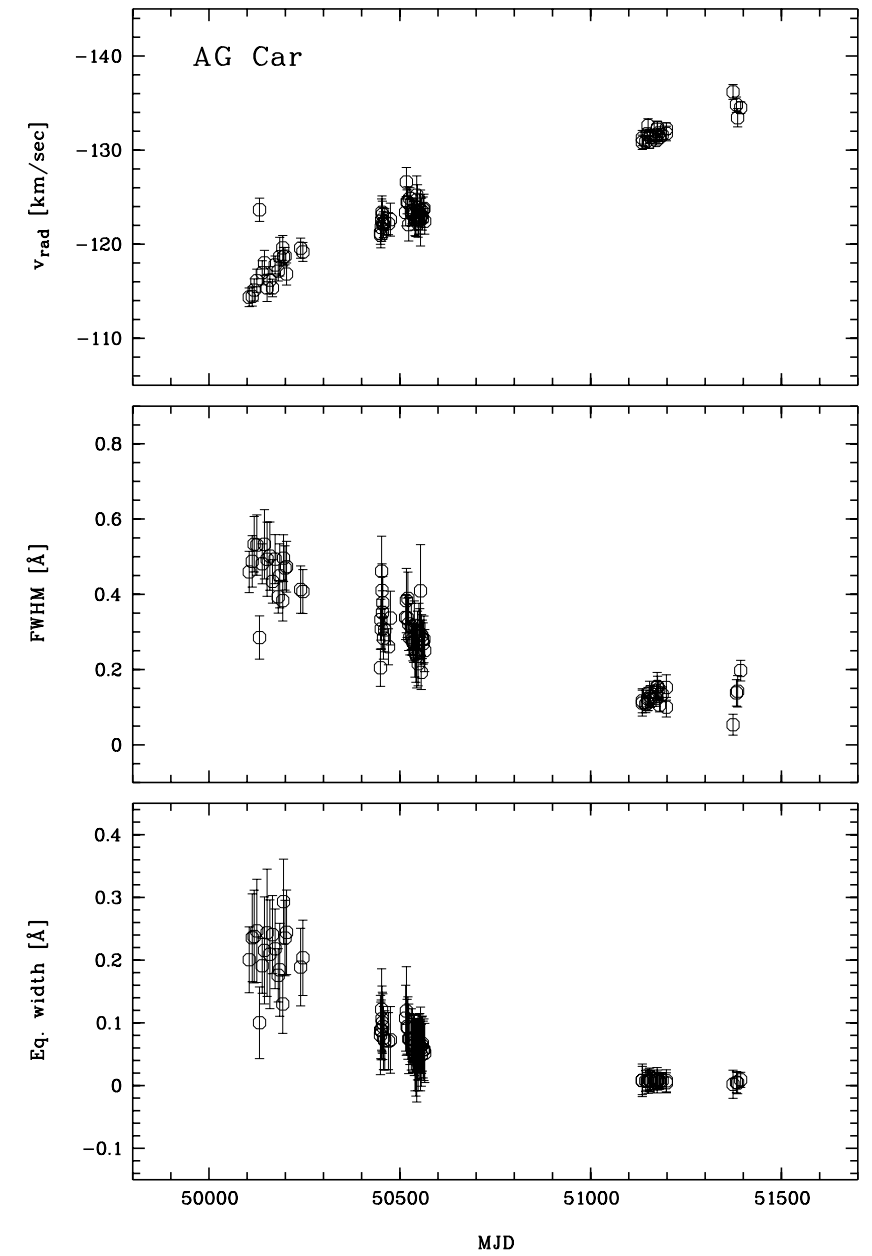

Fig. 12. Evolution of the fast component of the FeII $\lambda 5169$ line from 1996 to 1999.

line, which is apparently due to decreasing absorption at smaller expansion velocities.

A similar effect is also seen for the main absorption component at smaller expansion velocities. The center of gravity for this line shifts significantly to the blue (cf. Fig. 13). In addition, the emission component evolves into a peculiar, roughly triangular, shape.

In the HeI $\lambda 6678$ line, a high velocity absorption component is seen for the whole S Dor cycle of AG Car. It has the same velocity as the high-velocity component seen in the Balmer lines during the phase of brightening $\left(-265 \mathrm{~km} \mathrm{~s}^{-1}\right)$. In Fig. 14, we show the mean FLASH spectrum obtained in 1993 and the mean FEROs spectrum obtained in 1998/1999. More than 2000 days are covered by these observations and, taken together with the $\mathrm{H} \alpha$ and $\mathrm{H} \beta$ observations, the feature is visible for more than 3000 days. Apart from the high-velocity component, the HeI $\lambda 6678$ line has a stellar wind absorption component at low velocities, an additional absorption which is almost unshifted and an emission component.

The "un-shifted" component is particularly interesting, since most other lines only show blue-shifted absorption in the wind. The HeI lines are exceptional for this "un-shifted" absorption during maximum. We measured 


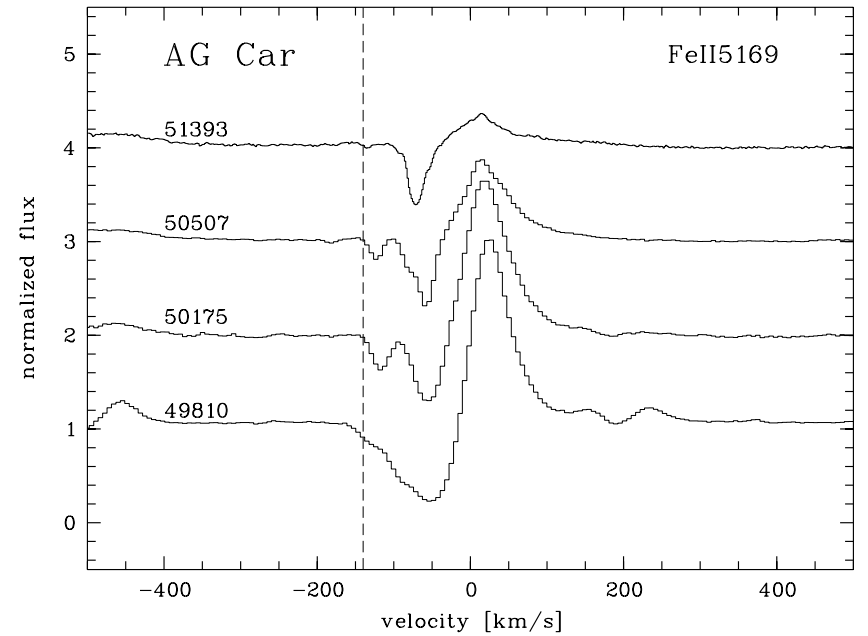

Fig. 13. Evolution of the FeII $\lambda 5169$ line during decrease from maximum light from 1995 to 1999 . The vertical line marks a heliocentric radial velocity of $-140 \mathrm{~km} \mathrm{~s}^{-1}$. The spectra are marked with MJD. Note that the blue-shifted absorption component has moved towards shorter wavelengths, but the edge velocity did not change. The main component also shifts towards higher blue-shifted velocities, while becoming narrower, and separates from the emission component.

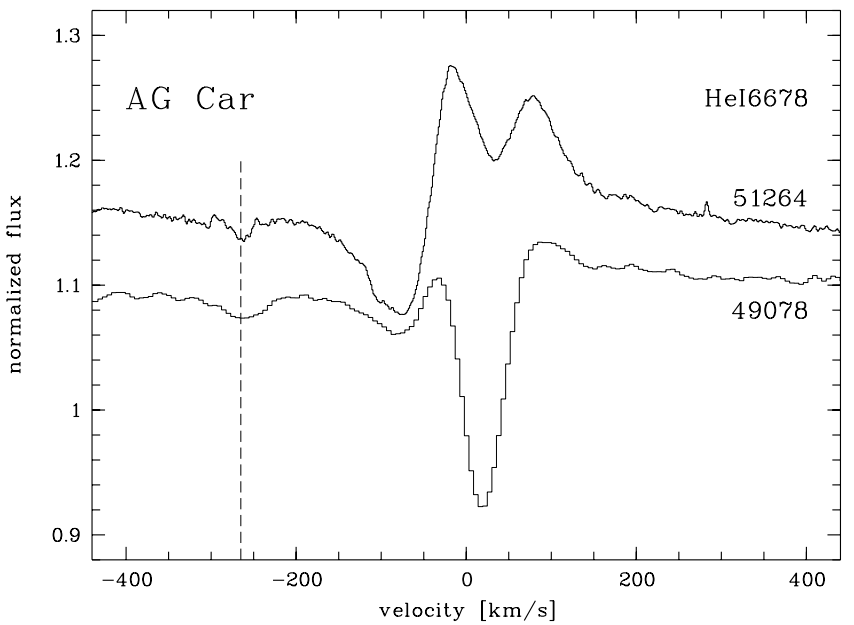

Fig. 14. The HeI $\lambda 6678$ line before and after maximum. The high velocity absorption feature is seen in both spectra and also during the full S Dor cycle. The spectra are marked with MJD. The vertical line marks a heliocentric radial velocity of $-265 \mathrm{~km} \mathrm{~s}^{-1}$. The line has become narrower with time, but the change in radial velocity is marginal.

the velocity of the "un-shifted" component for the part of the cycle, where these components are well visible and little influenced by emission. The results are presented in Fig. 15. The long-term trend is striking. Note that the velocities are partly significantly red-shifted with respect to the systemic velocity. The HeI $\lambda 5876$ line shows a similar behaviour in this component.

If the absorption features are identified with expanding shells, their long lifetimes imply very large distances. Assuming $270 \mathrm{~km} \mathrm{~s}^{-1}$ for the fastest component and a lifetime of 600 days, the travel distance for a shell would be

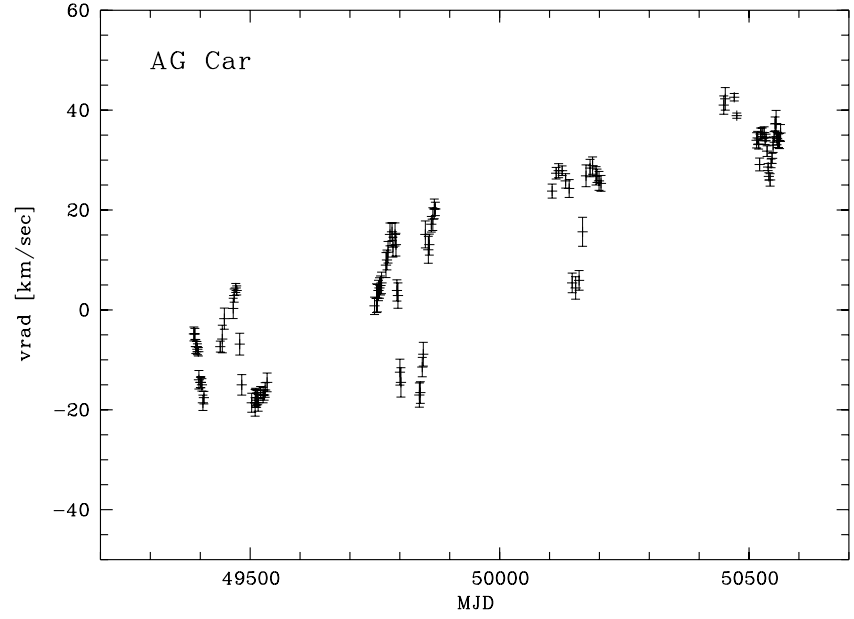

Fig. 15. The heliocentric radial velocity of the "un-shifted" absorption component and the stellar wind component of HeI $\lambda 6678$ during the S Dor cycle. A very long-term trend towards higher velocities is visible. Superposed on this trend are pulsation-like variations on a timescale of about two months.

$20000 R_{\odot}$, which is, depending on the stellar radius, of the order of $100 R_{*}$.

\subsection{Equivalent widths, line fluxes and line widths}

Since the physical conditions in the atmosphere and wind of AG Car change drastically during the S Dor cycle, the equivalent widths and line widths of most lines also show very pronounced changes. This is mainly an effect of the changing stellar effective temperature, wind velocity and wind density. Of particular interest are the Balmer lines, since they are little affected by ionization changes and their changes in equivalent width and line width may therefore indicate changes in mass-loss rate.

The strength of the emission component of $\mathrm{H} \alpha$ was measured by integrating from $6520 \AA$ to $6610 \AA$. These integration limits include the broad electron scattering wings. The $F W H M$ of $\mathrm{H} \alpha$ was also measured. The value was measured directly, i.e. it is not the result of a line fit but just the line width at half of the peak line intensity. The result of the measurements are shown in Figs. 16a and $\mathrm{b}$. Because of the strong changes in the continuum level, the changes in equivalent width do not directly correspond to changes in the line flux. The equivalent widths have been converted to fluxes by using the magnitudes from Fig. 1 and assuming a constant value of $V-R=0.5$. The data have been roughly calibrated assuming a value of $2.2 \times 10^{-9} \mathrm{erg} \mathrm{cm}^{-2} \mathrm{~s}^{-1} \AA^{-1}$ for an $R$-magnitude of 0.0 (Bessell 1979). Since it is known that LBVs have a redder color during maximum, the assumption of constant $V-R$ slightly underestimates the flux variations. Since the equivalent widths increase during the brightening (Fig. 16b), the line fluxes changes are stronger than the changes in equivalent width (Fig. 16c).

The equivalent width increases during maximum, but the peak does not coincide with the maximum brightness. 
The peak in equivalent width is reached around MJD = 50150 and the maximum emission line flux at about MJD $=50000$. The maximum visual brightness was reached much earlier, around MJD $=49700$. Note that the equivalent width changes of the $\mathrm{H} \alpha$ line do not follow the changes in line width (Fig. 16). Most of the change in flux is due to an increase of the peak flux. The FWHM changes very strongly in the early phase of the cycle and shows only minor changes later during maximum phase. It has a local maximum close to the maximum visual brightness.

\section{Modeling of $\mathrm{H} \alpha$}

In order to quantitatively investigate the mass-loss history of AG Car we modeled the $\mathrm{H} \alpha$ line for selected phases. We do not attempt a full spectroscopic analysis but our aim is to get an order of magnitude estimate of the rate. We base our computations on the Kiel code for spherically expanding atmospheres (Hamann \& Schmutz 1987; Wessolowski et al. 1988) that was expanded by Schmutz (1991) to treat line blanketing and further extended by Schaerer \& Schmutz (1994) to calculate the density structure from self consistent hydrodynamic wind solutions with the wind driven by radiation pressure. The full version of the model has been applied to AG Car by Schmutz (1997), who found that it was marginally possible to explain the wind of AG Car by radiation pressure. Here we simplify the computations by using a pre-defined velocity law instead of a calculated hydrodynamic solution. The reason for this approach is that it is very time consuming to perfom the iterations for a self-consisting hydrodynamical solution. In line with our aim to get only an estimate of the parameters, the atomic model is small, consisting of five levels of neutral hydrogen and 11 levels of neutral helium. Thus, including the ionized states, there are 13 levels.

The basic stellar parameters, $T_{\text {eff }}$ and $R_{*}$, are derived similar to Sect. 3.1, assuming a constant luminosity of $L=$ $10^{6} L_{\odot}$ but, in contrast to Sect. 3.1, using the emissivity of a black body instead of relation (1). Relation (1) was then obtained from the fluxes predicted by these models. In principle, we could iterate for better stellar parameters but for the present aim, an estimate of the mass loss rate, the additional effort is not worthwhile. As in Schmutz (1997) we adopt the helium abundance $N[\mathrm{He}]=0.29$, resulting from an analysis of a minimum spectra by Smith et al. (1994). We vary the mass loss rate and the velocity law to fit the observed $\mathrm{H}_{\alpha}$ line profile for selected dates. The results are given in Table 6 .

The profile of MJD $=48247$ (December 1990) can be reproduced satisfactorily with a $\beta$-law where $\beta=1$. A comparison with the hydrodynamically calculated law in Schmutz (1997), that was obtained for the same date, shows that the $\beta$-law approximates the velocity distribution around optical depth $\tau_{\mathrm{R}}=0.1$ but disagrees at larger distances from the photosphere in that the $\beta$-law faster approaches the terminal velocity. Consequently, with our approach here we underestimate the terminal velocity. We get $225 \mathrm{~km} \mathrm{~s}^{-1}$ instead of the $280 \mathrm{~km} \mathrm{~s}^{-1}$ determined by

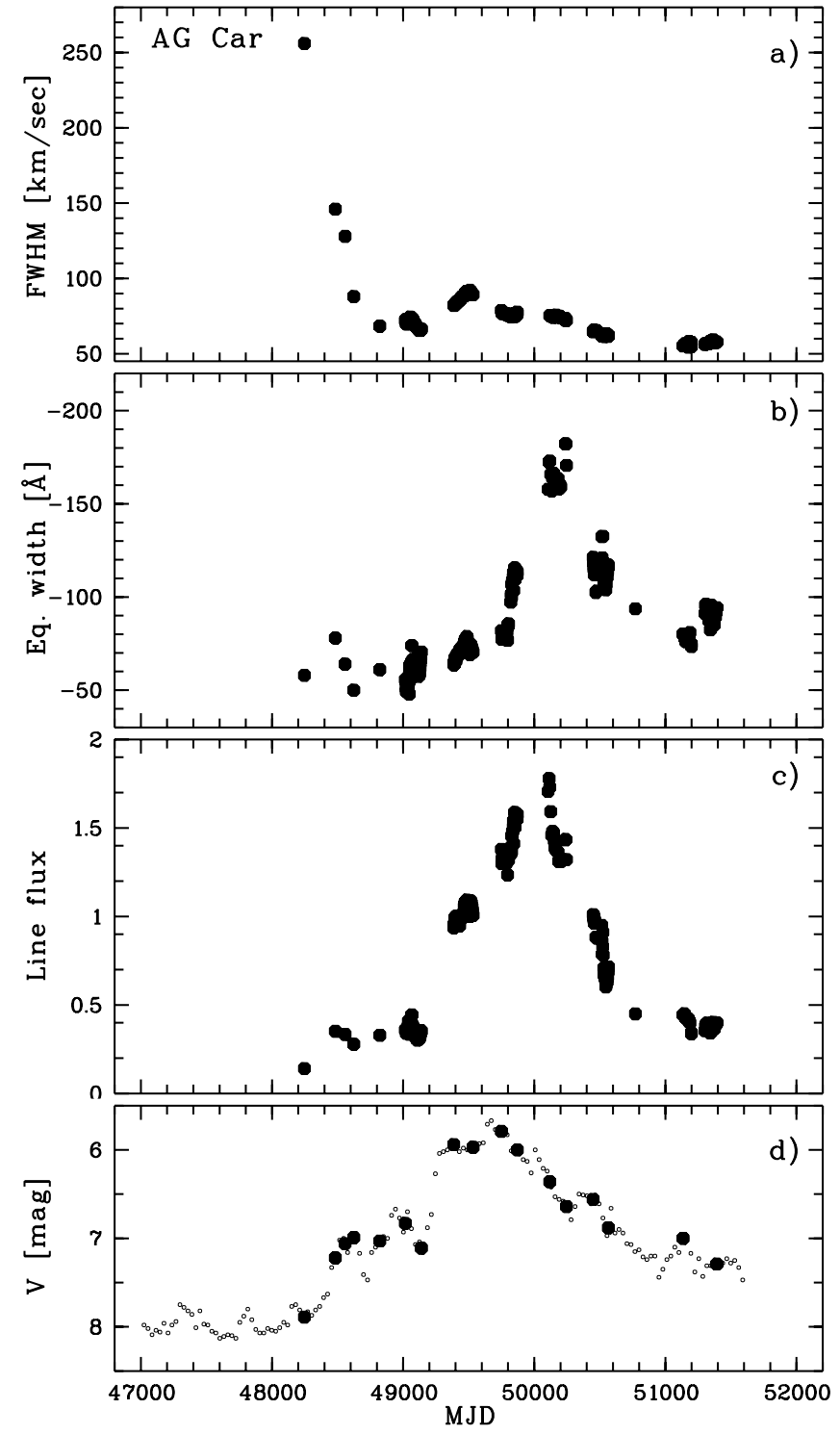

Fig. 16. $F W H M$, equivalent width and emission-line flux of $\mathrm{H} \alpha$ versus date. The visual light curve is plotted again to compare the time dependencies of the data. The measured equivalent widths have been converted to fluxes by using the magnitudes from Fig. 1. Flux units are $10^{-9} \mathrm{erg} \mathrm{cm}^{-2} \mathrm{~s}^{-1}$. In d), the dates where models have been computed are marked by filled symbols.

Schmutz (1997). Also the mass loss rate differs between the two analyses because the adopted stellar parameters are not identical: here we find $\log \dot{M}=-4.5$ whereas Schmutz (1997) determined -4.4. The difference of the two determinations gives a rough estimate of the accuracy of the present mass loss determination.

The observed $\mathrm{H} \alpha$ profiles after Dec. 1990 significantly differ in shape: their shape is much narrower, they have P Cygni absorptions, and there are broad line wings similar to those of the HeI lines mentioned in Sect. 3.3. These wings are only partly due to electron scattering. We interpret these wings partly as remnants of the former wind properties of AG Car that once had a higher terminal velocity. Here the reality is obviously more complicated than 
our model that assumes a steady state situation. In order to better fit the line profiles we modified the $\beta$-law and added a term that is proportional to the radius. The velocity laws are given in Eqs. (2)-(4) and in Fig. 18 we display a few selected line profile fits. In principle, if we want to take the wind history into account we should also allow for varying mass loss rates in the past. However, as said already, we like to keep the modeling as simple as possible and therefore, each model is calculated with a given mass loss rate.

The inclusion of a linear term leads to profiles that improve the line fits considerably. In Fig. 17 we compare the resulting line profiles using a $\beta$-law and the law of Eq. (2). The comparison with the observations of MJD = 48822 demonstrates that the unsaturated absorption component as well the line wings are much better fitted with Eq. (2). It should be noted that both calculations predict relatively strong electron scattering wings that agree well with the observed profile. However, the electron scattering wings extend far beyond the wavelength range shown in Fig. 17.

The velocity laws $2-4$ formally have no terminal velocity. This is in agreement with reality where the wind of a parameter combination for a given date was not blowing long enough to reach terminal velocity. However, the terminal velocity of the $\beta$-law term gives an estimate of the velocity characteristics of the regions close to the photosphere and therefore, this number allows an estimate of the terminal velocity of the wind belonging to the analyzed date at least in a first approximation, similar to the result discussed above, where the terminal velocity that we determined for Dec. 1990 was also not far from the observed value.

As a general result, our line profiles predict broader P Cygni absorptions than observed. Therefore, from the model point of view, the features that were discussed in Sect. 3.4 as discrete absorption components are not discrete absorptions but instead, the emission between them are discrete emissions. Such emissions, or "missing absorptions", indicate lower densities at the corresponding expanding velocities. It looks as if the faster wind of the past, that we are approximating with the linear term, was interrupted for a while or, that the wind changed abruptly from a faster terminal velocity to a slower one, leading to a shell of lower density between the two states.

Selected results of our model fits for a few phases are shown in Fig. 18.

$$
\begin{aligned}
& v=\left\{\begin{array}{ll}
v_{\infty}(1-1 / r)^{8}+(r-1) & \text { if } r<301 \\
v_{\infty}(1-1 / r)^{8}+300+(r-301) / 8 & \text { if } r>301
\end{array}\right\} \\
& v=\left\{\begin{array}{ll}
v_{\infty}(1-1 / r)^{8}+(r-1) & \text { if } r<71 \\
v_{\infty}(1-1 / r)^{8}+70+(r-71) / 10 & \text { if } r>71
\end{array}\right\} \\
& v=\left\{\begin{array}{ll}
v_{\infty}(1-1 / r)^{8}+(r-1) / 3 & r<151 \\
v_{\infty}(1-1 / r)^{8}+50+(r-151) / 10 & r>151
\end{array}\right\} .
\end{aligned}
$$

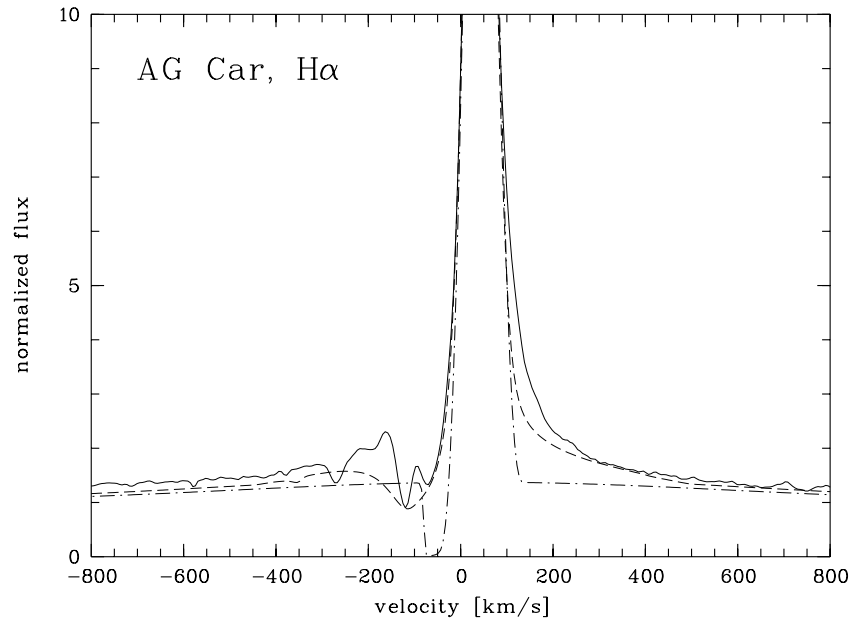

Fig. 17. $\mathrm{H} \alpha$ models compared with the observations for two velocity laws. The dash-dotted model was computed with a $\beta$-type velocity law, while the dashed profile in addition has a linear term as in Eq. (2). The latter velocity law produces much stronger line wings and also fits the unsaturated absorption much better.

Table 6. Summary of model parameters. $T_{\text {eff }}$ is given in $10^{3} \mathrm{~K}$ and $R$ in solar radii. Note that $v_{\infty}$ is not the terminal velocity of the wind but only a parameter in the velocity law. "shift" denotes the systemic velocity (in $\AA$ ) derived from the $\mathrm{H} \alpha$ fit. Values marked with ${ }^{1}$ are estimated only from models computed previously.

\begin{tabular}{llrlllll}
\hline Model & MJD & $T_{\text {eff }}$ & $R$ & $v_{\infty}$ & $\dot{M}$ & shift & Eq. \\
\hline $\mathrm{a}$ & 48247 & 24.06 & 58 & 225 & -4.48 & 0.7 & $\beta=1$ \\
$\mathrm{~b}$ & 48483 & 18.47 & 99 & 150 & -4.17 & 0.6 & Eq. (2) \\
$\mathrm{c}$ & 48557 & 17.23 & 114 & 170 & -4.30 & 0.6 & Eq. (2) \\
$\mathrm{d}$ & 48624 & 16.70 & 121 & 100 & -4.45 & $0.54^{1}$ & Eq. (2) \\
1 & 48822 & 16.97 & 117 & 71 & -4.53 & 0.54 & Eq. (2) \\
2 & 49016 & 15.62 & 140 & 85 & -4.46 & 0.6 & Eq. (2) \\
3 & 49139 & 17.59 & 109 & 56 & -4.54 & 0.5 & Eq. (2) \\
4 & 49386 & 9.62 & 365 & 140 & -4.10 & 0.4 & Eq. (2) \\
5 & 49534 & 9.78 & 354 & 130 & -4.05 & 0.4 & Eq. (3) \\
6 & 49749 & 8.55 & 463 & 95 & -3.93 & $0.5^{1}$ & Eq. (3) \\
7 & 49871 & 9.99 & 338 & 70 & -3.95 & $0.5^{1}$ & Eq. (3) \\
8 & 50118 & 12.37 & 222 & 40 & -3.83 & $0.5^{1}$ & Eq. (3) \\
9 & 50245 & 14.29 & 166 & 33 & -3.80 & $0.5^{1}$ & Eq. (3) \\
10 & 50449 & 13.72 & 180 & 35 & -4.00 & $0.45^{1}$ & Eq. (3) \\
11 & 50565 & 15.98 & 134 & 33 & -4.10 & 0.45 & Eq. (3) \\
12 & 51135 & 16.76 & 120 & 48 & -4.50 & 0.45 & Eq. (4) \\
13 & 51392 & 19.00 & 94 & 47 & -4.50 & 0.45 & Eq. (4) \\
\hline
\end{tabular}

\section{Variability on shorter timescales}

\subsection{Hel lines}

The most pronounced variations of AG Car on shorter timescales are shown by the HeI lines. These lines show a very peculiar behaviour over the $\mathrm{S}$ Dor cycle. During minimum phase, the HeI lines show classical P Cyg profiles. During maximum phase, the profiles are much more complicated. Dynamical spectra in the HeI $\lambda 6678$ and HeI $\lambda 5876$ lines are shown in Figs. 19, 20. We selected the HeI $\lambda 6678$ line for presentation of the 1993 data, since the 

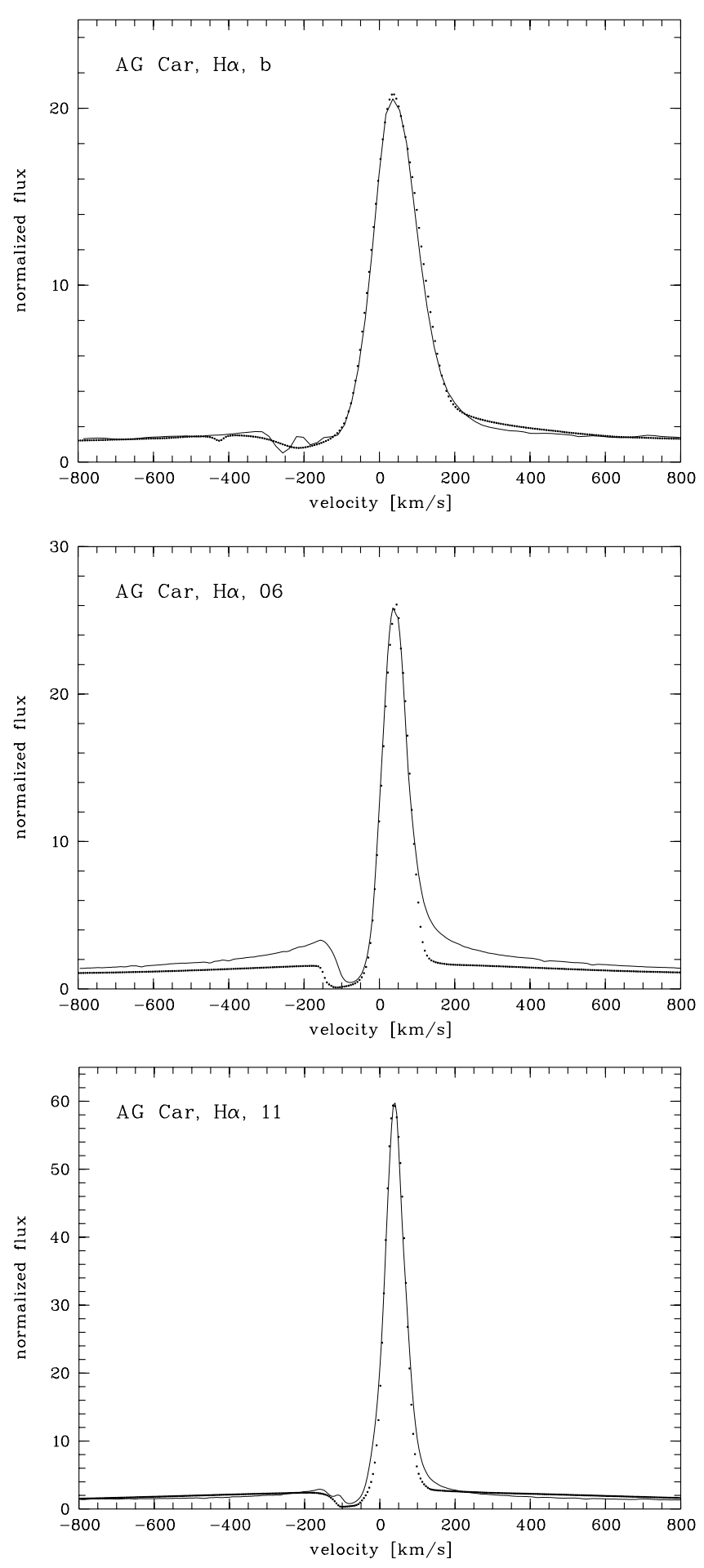

Fig. 18. Selected $\mathrm{H} \alpha$ profiles and the corresponding model profiles. The curves are labeled with the model number given in Table 6.

line HeI $\lambda 5876$ is disturbed by a CCD defect in that years' run. The HeI $\lambda 6678$ line, on the other hand, is blended with a line near maximum light, so that we show HeI $\lambda 5876$ instead. The blending FeII line does not contribute significantly in 1993.

The HeI lines show a central absorption which may be accompanied by a blue and/or a red emission component. In addition, a blue-shifted absorption component may be

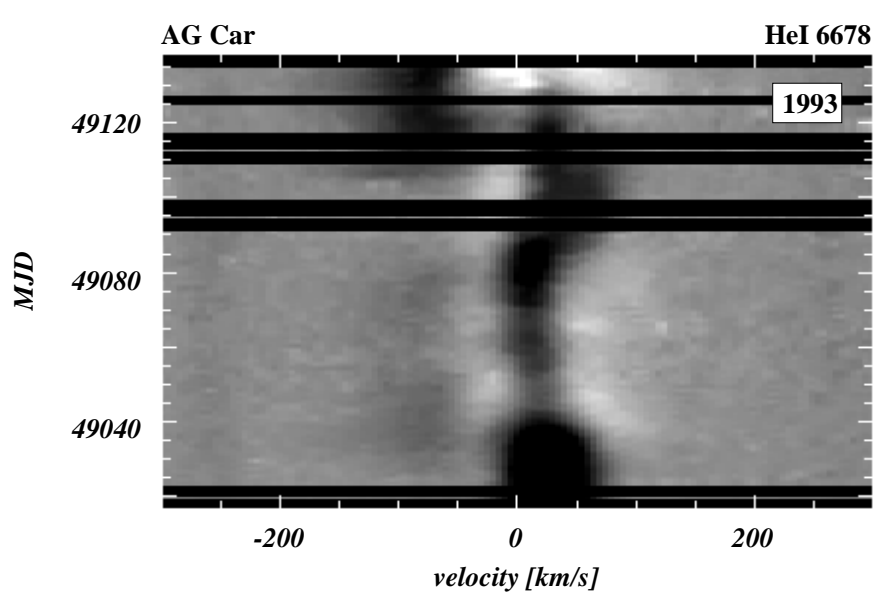

Fig. 19. Dynamical spectrum of AG Car in the HeI $\lambda 6678$ line obtained in 1993. The spectra in this season were obtained on the slow rise to maximum, but in a short phase of decreasing brightness.

present during increase to - and decrease from maximum (Figs. 20, 19, 4). During these phases, the profile shape is especially complicated. In addition, very broad and shallow emission wings are observed in the strongest HeI lines (Fig. 8).

The most obvious form of variability are variations of the radial velocity of the central absorption (cf. Figs. 20 and 15). The timescale of these variations is of the order of about two months. Apart from that, there are occasionally much faster variations, down to timescales of only a few days. An example of fast variability can be seen in Fig. 19, where a fast blue-shifted component appears within a few days. A long-term trend is also visible (Fig. 15). The central absorption component, which is near the systemic velocity, moves towards the red and is significantly red-shifted at the end of the time series. The velocity is close to the system velocity around visual maximum. If this absorption component is of photospheric origin, the red-shift has to be interpreted as a shrinking photosphere. In principle this behaviour is very similar to what is expected from a simple model for S Dor cycles: The radius increases before maximum and decreases after maximum. The expected velocities are a only few $\mathrm{km} \mathrm{s}^{-1}$, however. The observed velocities are significantly larger than that. However, the observation of red-shifted lines originating close to the star clearly indicates that the continuum forms in a photosphere which is not always expanding but is more or less static with superimposed short-term oscillations and a long-term trend which is possibly related to the $\mathrm{S}$ Dor cycle.

The radial-variations with a time scale of about two months are particularly interesting, because they appear to be quite regular. They look pulsation-like but could also be due to other processes such as e.g. rotation. A similar time scale seems not to be present in the light variations. AG Car does show micro-variations, but the period is of the order of 10-14 days only (van Genderen et al. 1988, 1990), with no strong dependence on the brightness level. 
It should be noted, however, that the maximum phase of AG Car has not been sufficiently well observed to exclude a similar photometric period of about two months. Similar periods have been seen in other LBVs during maximum and called the "100 $d$ type variations" by van Genderen (2001). The characteristics of the spectroscopic variations, including the appearance of blue and red emission and the occasional sudden appearance of fast blue-shifted absorption components are reminiscent of the $\mathrm{H} \alpha$ line variability seen in normal BA-type supergiants (Kaufer et al. 1996a, 1996b). For the latter aspect cf. Fig. 19 with Fig. 2 of Kaufer et al. (1996a). As in the BA-type supergiants, the timescale does not allow to distinguish between rotation and pulsation. The characteristics are more easily explained by a rotating and flattened envelope, however.

\section{Discussion and conclusion}

While the basic variability during the S Dor cycle seems qualitatively understood, a number of spectroscopic observations is not fully understood.

The appearance of multiple discrete absorption components in LBVs is spectacular. Although discrete absorption components, and more generally wind structures, are commonly observed in normal hot stars (e.g. Fullerton et al. 1997; Prinja et al. 2001), the effect is much more pronounced in AG Car. The time scales are also much longer than for normal hot stars. While in normal hot stars the time scales are days to weeks, they can reach several years in AG Car. The time scales seem to be of the order of the length of the S Dor cycle, rather than of the order of the kinematic timescale of the wind or the rotational timescale. Therefore it seems plausible that a completely different mechanism is involved in LBVs.

In AG Car, the line splitting is especially common during the beginning of the rise to visual maximum. A similar behaviour was observed in the LBV R 127 of the LMC (Stahl et al. 1983). This line-splitting has been explained by multiple discrete expanding shells. However, this explanation is difficult to sustain. The structures are very long-lived and it appears that the splitting is due to missing absorption at intermediate velocities, and not extra absorption due to shells. Possibly some of the features just reflect the changing stellar wind velocity of the underlying star. In a phase of decreasing stellar wind velocity, a detached shell, which still shows the higher wind velocity of the pre-maximum wind, could form. Other line-splitting events could be due to ionization structures.

In contrast to the general appearance of the spectrum, the quantitative wind properties do not follow the light curve very closely. Assuming an increase of the stellar radius of a factor of 10 during maximum, we expect the escape velocity to decrease by a factor of 3.3. For normal stellar winds of hot stars, $v_{\infty} / v_{\text {esc }}$ is expected to decrease with decreasing temperature (Lamers et al. 1995), so that the decrease in stellar wind velocity around maximum is expected to be even larger than that. The observed decrease of the velocity of the stellar wind is smaller than

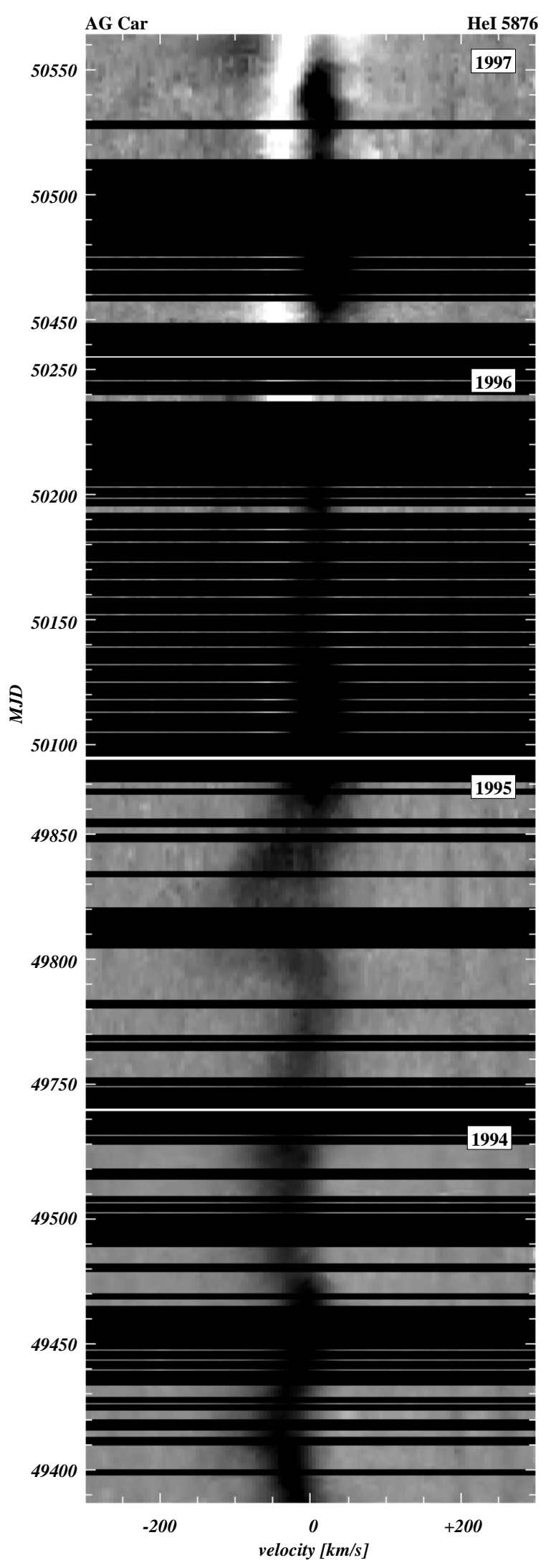

Fig. 20. Dynamical spectrum of AG Car in the HeI $\lambda 5876$ line obtained from 1994 to 1997 . The data cover the maximum phase and the slow decrease after the maximum. 
expected, but due to the unknown effect of turbulent broadening on the line profiles and other uncertainties, we cannot exclude that the terminal velocity of the stellar wind changes in accordance with the expectation of the theory of radiation-driven winds.

The equivalent width of $\mathrm{H} \alpha$ increases with visual brightness. Most of the increase seems to occur at temperatures $T_{\text {eff }}<15000 \mathrm{~K}$. However, the equivalent width peaks long after the maximum light. This means that at the same brightness level after maximum, the line flux is up to five times larger than at the same brightness before maximum. If radius and effective temperature are a function of the brightness only, this implies that the emissionline flux is not only a function of radius and temperature, which is expected for a radiation-driven stellar wind. According to our modeling, the derived mass-loss rates are approximately proportional to the emission-line flux. An increase of the mass-loss rate by about a factor 5 during the $\mathrm{S}$ Dor cycle is therefore indicated by our modeling. A possible explanation would be a higher luminosity during the decrease from maximum due to the release of gravitational energy (Lamers 1995). The expected effect is much smaller than what we observe, however.

The inferred increase in mass-loss rate may partly be an artefact of our modeling, which assumes a stationary stellar wind. In reality, the wind expansion time scale is not small compared to the time scale for changes at the base of the stellar wind, at least for the shallow stellar-wind velocity laws which we derive. This means that mass can accumulate around the star during phases of small stellar wind velocities. This accumulated matter contributes to the $\mathrm{H} \alpha$ emission and could increase the inferred mass-loss rates, even without any change in massloss. The observed delay of the line-flux maximum with respect to the light curve could be due to this accumulation of matter around the star. Because of the uncertainties in the wind velocity law, the stellar wind time scale is very uncertain, however.

The mass-loss rate also seems to increase slightly at the beginning of the cycle, but returns to its minimum phase value afterwards. We therefore have no evidence for a strong increase in mass-loss rate in the early phase of the cycle. This argues against models which link origin of the S Dor cycle to changes in mass-loss rate (e.g. Davidson 1987).

The inverse P Cygni profiles and the double-peak profiles observed in the the HeI lines of AG Car are reminiscent of the $v / r$-variations observed in classical Be stars. The timescale of the variations is longer than in Be stars, but this may be due to the larger stellar radius and therefore longer rotational time scale. Inverse $\mathrm{P}$ Cyg profiles have been observed a few times before in LBVs (Wolf \& Stahl 1990). However, AG Car is the first case where we also observe double-peak profiles, and where the emission lines are clearly red- and blue-shifted. The observation of red-shifted absorption lines after maximum indicates that the continuum-forming layers are not strongly expanding but at least at some phase are even shrinking. It is in fact not clear if we see an expanding photosphere at all. It appears that the photosphere is more or less static with some superimposed oscillations. It is therefore unlikely that an optically thick stellar wind causes the radius increase during S Dor cycles.

Acknowledgements. We thank the European Southern Observatory (ESO) for the generous allocation of observing time and the staff at La Silla for the support in the installation of our Heros instrument at the telescope. We acknowledge the use of the SIMBAD database (CNRS data center, Strasbourg). This research has made use of NASA's Astrophysics Data System (ADS) Abstract Service. This work was supported by the Deutsche Forschungsgemeinschaft (Wo 296, 9-1). I. J and J. K. thank the Hungarian Scientific Research Fund (OTKA) for support.

\section{References}

Bateson, F. M. 1988-2000, RASNZ Var. Star Sect. Circ.

Bernat, A. P., \& Lambert, D. L. 1978, PASP, 90, 520

Bessell, M. S. 1979, PASP, 91, 589

Bowen, I. S. 1960, ApJ, 132, 1

Davidson, K. 1987, ApJ, 317, 760

de Koter, A. 1997, in Luminous Blue Variables: Massive Stars in Transition, ed. A. Nota, \& H. J. G. L. M. Lamers, ASP Conf. Ser., 120 (ASP), 66

de Koter, A., Lamers, H. J. G. L. M., \& Schmutz, W. 1996, A\&A, 306, 501

Fullerton, A. W., Massa, D. L., Prinja, R. K., Owocki, S. P., \& Cranmer, S. R. 1997, A\&A, 327, 699

Guzik, J., Cox, A. N., Despain, K. M., \& Soukop, M. S. 1998, in Variable and Non-spherical Stellar Winds in Luminous Hot Stars, ed. B. Wolf, O. Stahl, \& A. W. Fullerton, IAU Colloq. 169 (Springer), 337

Hamann, W. R., \& Schmutz, W. 1987, A\&A, 174, 173

Hoekzema, N. M., Lamers, H. J. G. L. M., \& van Genderen, A. M. 1992, A\&A, 257, 118

Humphreys, R. M., Lamers, H. J. G. L. M., Hoekzema, N., \& Cassatella, A. 1989, A\&A, 218, L17

Kaufer, A., Stahl, O., Tubbesing, S., et al. 2000, in Optical and IR Telescope Instrumentation and Detectors, ed. A. F. Moorwood, Proc. of the SPIE, No. 4008 (SPIE)

Kaufer, A., Stahl, O., Wolf, B., et al. 1996b, A\&A, 314, 599

Kaufer, A., Stahl, O., Wolf, B., et al. 1996a, A\&A, 305, 887

Lamers, H. J. G. L. M. 1995, in Astrophysical applications of stellar pulsation, ed. R. S. Stobie, \& P. Whitelock, ASP Conf. Ser., 83 (ASP), 176

Lamers, H. J. G. L. M., Snow, T. P., \& Lindholm, D. M. 1995, ApJ, 455, 269

Leitherer, C. 1997, in Luminous Blue Variables: Massive Stars in Transition, ed. A. Nota, \& H. J. G. L. M. Lamers, ASP Conf. Ser., 120 (ASP), 58

Leitherer, C., Allen, R., Altner, B., et al. 1994, ApJ, 428, 292

Leitherer, C., Appenzeller, I., Klare, G., et al. 1985, A\&A, 153, 168

Leitherer, C., Schmutz, W., \& Abbott, D. C. 1989, ApJ, 346, 919

Mandel, H. 1988, in The impact of very high $S / N$ spectroscopy on stellar physics, ed. G. C. de Strobel, \& M. Spite, Proc. of the IAU Symp., 132 (Kluwer), 9 
Nota, A., Livio, M., Clampin, M., \& Schulte-Ladbeck, R. 1995, ApJ, 448, 788

Ponz, D., \& Brinks, E. 1986, The ESO Messenger, 43, 31

Prinja, R. K., Barlow, M. J., \& Howarth, O. D. 1990, ApJ, 361,607

Prinja, R. K., Stahl, O., Kaufer, A., et al. 2001, A\&A, 367, 891

Schaerer, D., \& Schmutz, W. 1994, A\&A, 288, 231

Schmutz, W. 1991, in Stellar Atmospheres: Beyond Classical Models, ed. L. Crivellari, I. Hubeny, \& D. G. Hummer, NATO ASI Ser. C, 341 (Kluwer, Dordrecht), 191

Schmutz, W. 1997, in Luminous Blue Variables: Massive Stars in Transition, ed. A. Nota, \& H. J. G. L. M. Lamers, ASP Conf. Ser., 120 (ASP), 143

Schulte-Ladbeck, R. E., Clayton, G. C., Hillier, D. J., Harries, D. J., \& Howarth, I. D. 1994, ApJ, 429, 846

Shore, S. N., Altner, B., \& Waxin, I. 1996, AJ, 112, 2744

Smith, L. J., Crowther, P. A., \& Prinja, R. K. 1994, A\&A, 281, 833

Smith, L. J., Stroud, M. P., Esteban, C., \& Vilchez, J. M. 1997, MNRAS, 290, 265

Spoon, H. W. W., de Koter, A., Sterken, C., Lamers, H. J. G. L. M., \& Stahl, O. 1994, A\&AS, 106, 141

Stahl, O. 1986, A\&A, 164, 321

Stahl, O. 1997, in Luminous Blue Variables: Massive Stars in Transition, ed. A. Nota, \& H. J. G. L. M. Lamers, ASP Conf. Ser., 120 (ASP), 100

Stahl, O. 1998, in Variable and Non-spherical Stellar Winds in Luminous Hot Stars, ed. B. Wolf, O. Stahl, \& A. W. Fullerton, IAU Colloq., 169 (Springer), 251

Stahl, O. 2001, in Eta Carinae and Other Mysterious Stars: the Hidden Opportunities of Emission-Line Spectroscopy, ASP Conf. Ser., (ASP)
Stahl, O., Kaufer, A., Rivinius, T., et al. 1996, A\&A, 312, 539

Stahl, O., Kaufer, A., \& Tubbesing, S. 1999, in Optical and infrared spectroscopy of circumstellar matter, ASP Conf. Ser., 188 (ASP), 331

Stahl, O., Mandel, H., Wolf, B., et al. 1993, A\&AS, 99, 167

Stahl, O., \& Wolf, B. 1982, A\&A, 110, 27

Stahl, O., Wolf, B., Klare, G., et al. 1983, A\&A, 127, 49

Stahl, O., Wolf, B., Klare, G., Jüttner, A., \& Cassatella, A. 1990, A\&A, 228, 379

Stothers, R. B. 1999, ApJ, 516, 366

Stothers, R. B., \& Chin, C. 1995, ApJ, 451, L61

Thackeray, A. D. 1950, MNRAS, 110, 524

Thackeray, A. D. 1977, MNRAS, 180, 95

van Genderen, A. M. 1979, A\&AS, 38, 381

van Genderen, A. M. 2001, A\&A, 366, 508

van Genderen, A. M., Sterken, C., \& de Groot, M. 1997, A\&A, 318,81

van Genderen, A. M., Thé, P. S., Augusteijn, T., et al. 1988, A\&AS, 74, 467

van Genderen, A. M., Thé, P. S., Heemskerk, M., et al. 1990, A\&AS, 82, 189

Viotti, R., Altamore, A., Barylak, M., Gilmozzi, R., \& Rossi, C. 1984, in Future of UV Astronomy based on Six Years of IUE research, NASA CP-2349, 231

Wessolowski, U., Schmutz, W., \& Hamann, W. R. 1988, A\&A, 194, 160

Whitelock, P. A., Carter, B. S., Roberts, G., Whittet, D. C. B., \& Baines, D. W. T. 1983, MNRAS, 205, 577

Wolf, B. 1989, A\&A, 217, 87

Wolf, B., Appenzeller, I., \& Stahl, O. 1981, A\&A, 103, 94

Wolf, B., \& Stahl, O. 1982, A\&A, 112, 111

Wolf, B., \& Stahl, O. 1990, A\&A, 235, 340 